Journal for ImmunoTherapy of Cancer

\section{Interferon gamma inhibits CXCL8- CXCR2 axis mediated tumor-associated macrophages tumor trafficking and enhances anti-PD1 efficacy in pancreatic cancer}

Mingjie Zhang (D) , ${ }^{1}$ Lifeng Huang, ${ }^{2}$ Guoping Ding, ${ }^{3}$ Huilian Huang, ${ }^{4}$ Guoliang Cao, ${ }^{1}$ Xu Sun, ${ }^{1}$ Neng Lou, ${ }^{1}$ Qiang Wei, ${ }^{1}$ Tao Shen, ${ }^{3}$ Xiaodong Xu, ${ }^{3}$ Liping Cao, ${ }^{3}$ Qiang Yan ${ }^{1}$
To cite: Zhang M, Huang L, Ding G, et al. Interferon gamma inhibits CXCL8-CXCR2 axis mediated tumor-associated macrophages tumor trafficking and enhances anti-PD1 efficacy in pancreatic cancer. Journal for ImmunoTherapy of Cancer 2020;8:e000308. doi:10.1136/ jitc-2019-000308

- Additional material is published online only. To view please visit the journal online (http://dx.doi.org/10.1136/jitc2019-000308).

MZ, LH and GD contributed equally.

Accepted 09 January 2020

Check for updates

(c) Author(s) (or their employer(s)) 2020. Re-use permitted under CC BY-NC. No commercial re-use. See rights and permissions. Published by BMJ.

For numbered affiliations see end of article.

\section{Correspondence to \\ Dr Mingjie Zhang; \\ zmj@hzhospital.com}

Professor Liping Cao; caolipingzju@zju.edu.cn

Dr Qiang Yan; yanqiangdoc@hotmail.com

\section{ABSTRACT}

Background Pancreatic cancer (PC) is a common malignancy of the digestive system and is characterized by poor prognosis and early metastasis. Tumor immune escape plays an important role in PC progression. Programmed death 1 (PD1) blockade therapy is a promising treatment for patients with $\mathrm{PC}$, but is yet to achieve significant clinical effects so far. Interferon gamma (IFN- $\gamma$ ) is a soluble dimeric cytokine that is closely associated with tumor immune surveillance and cytotoxicity. IFN- $\gamma$ suppresses a variety of tumor-derived cytokines in PC, such as CXCL8. In the present study, we investigated the therapeutic efficacy of combined anti-PD1 and IFN- $\gamma$ treatment in PC.

Methods BxPC- 3 and Panc- 1 human PC cell lines were used to construct a murine PC model. Blood samples $(n=44)$ and surgical resection specimens $(n=36)$ from human patients with PC were also collected. $\chi^{2}$ test, twotailed unpaired t-test or Kaplan-Meier survival analysis was used to calculate $p$ values.

Results PD1/PD-L1 signaling was overexpressed in PC tumor-bearing mice. Anti-PD1 prevented tumor growth if initiated early after tumor inoculation; however, delayed antiPD1 treatment showed limited benefit. Murine PC model had a preferential expansion of CXCR2 ${ }^{+} \mathrm{CD} 68^{+}$macrophages, and these cells showed an immunosuppressive nature (M2 polarization). PC tumors overexpressed CXCL8 and tumor-derived CXCL8 deficiency prohibited the trafficking of $\mathrm{CXCR}^{+} \mathrm{CD}^{+} 8^{+}$macrophages. IFN- $\gamma$ suppressed the expression of tumor-derived CXCL8, and combined with IFN- $\gamma$ treatment, delayed anti-PD1 treatment showed significant antitumor effects. Thus, we conclude that murine $\mathrm{CXCR}^{+} \mathrm{CD}^{+} 8^{+}$macrophages traffic to $\mathrm{PC}$ tumors by tumorderived CXCL8 and mediate local immunosuppression, which limits the efficacy of PD1 blockade therapy. IFN- $\gamma$ suppresses tumor-derived CXCL8 and inhibits the tumor trafficking of $\mathrm{CXCR}^{+} \mathrm{CD} 8^{+}$macrophages by blocking the CXCL8-CXCR2 axis to enhance anti-PD1 efficacy. Human PC also produces high levels of CXCL8. Patients with PC present elevated CXCR2 expression on peripheral and tumor-infiltrating $\mathrm{CD}^{+} 8^{+}$ macrophages, which are associated with advanced tumor stage and poor prognosis.
Conclusion Our findings suggest that IFN- $\gamma$ is a translatable, therapeutic option to improve the efficacy of PD1 blockade therapy by preventing trafficking of $\mathrm{CXCR}^{+} \mathrm{CD}^{+} 8^{+}$macrophages via blocking the CXCL8CXCR2 axis.

\section{BACKGROUND}

Pancreatic cancer (PC) is one of the most lethal types of cancer and carries a dire prognosis with a 5-year survival rate of only $3 \%-6 \% .^{12} \mathrm{PC}$ is characterized by immune tolerance and immunotherapeutic resistance. ${ }^{3}$ Programmed death 1 (PD1) signaling of host $\mathrm{T}$ cells in the tumor is a major mechanism of tumor immune escape. ${ }^{4-7}$ Tumor expression of programmed death-ligand 1 (PD-L1) inhibits tumor-reactive T cells in PC animal models, and several clinical studies show a close relationship between tumor expression of PD-L1 and adverse outcomes in patients with PC. ${ }^{8-10}$ Although accumulating evidence suggests that the PD1/PD-L1 axis plays an important role in PC immune escape, clinical application of anti-PD1 therapy alone shows optimistic antitumor effects in a minority of cases, ${ }^{11}$ suggesting that other mechanisms also contribute to immune evasion in patients with PC.

Tumor-associated macrophages (TAMs) constitute the most important component of the tumor microenvironment (TME), ${ }^{12} 13$ which can be polarized into a classically activated (M1) or alternatively activated (M2) status depending on different stimuli. ${ }^{14} 15$ M1 macrophages are tumoricidal, whereas M2 macrophages facilitate tumor progression. ${ }^{1617}$ Expansion of M2 TAMs is another mechanism of tumor immune escape and a number of approaches have been investigated 
to reprogram the polarization of TAMs to an immunogenic phenotype ${ }^{18-20}$ which may hold promise in the field of tumor immune therapy in patients with PC.

Interferon gamma (IFN- $\gamma$ ) is a dimeric soluble cytokine and is involved in tumor immune surveillance and cytotoxicity. ${ }^{21}$ IFN- $\gamma$ exerts its immunostimulatory and immunomodulatory effects primarily by activating the Janus kinase 1 (JAK1) and signal transducer and activator of transcription 1 (STAT1) signaling pathways. ${ }^{22}$ IFN- $\gamma$ is an important activator of macrophages and a key stimulus for inducing M1 polarization of macrophages. ${ }^{14} 23$ Our previous study proved that IFN- $\gamma$ suppresses a variety of tumor-derived cytokines, such as CXCL8, in PC tumors. ${ }^{24}$ CXCL8 is an important pro-inflammatory chemokine and mediates its signals via extracellular binding to two $\mathrm{G}$ protein-coupled receptors, C-X-C chemokine receptor type 1 (CXCR1) and C-X-C chemokine receptor type 2 (CXCR2) ${ }^{25}$ TAMs widely express CXCR1 and CXCR2 ${ }^{13}{ }^{26}$ so the CXCL8-CXCR1/2 axis appears critical for TAMs mobilization and recruitment in the PC TME and plays a crucial role in tumor immune escape. ${ }^{182728}$

Based on the aforementioned data, we could reasonably hypothesize that IFN- $\gamma$ may prohibit the tumor trafficking and infiltration of CXCR1/2-expressing TAMs by downregulating tumor-derived CXCL8, thus reducing resistance to PD1 blockade. In the present study, we report that murine $\mathrm{PC}$ induces the preferential expansion of CXCR $2^{+} \mathrm{CD}_{6} 8^{+}$macrophages, and these immunosuppressive cells traffic to the PC tumor bed by tumor-derived CXCL8 and mediate local immunosuppression to limit the efficacy of PD1 blockade therapy. IFN- $\gamma$ suppresses the expression of PC tumor-derived CXCL8, thus, inhibiting the trafficking of $\mathrm{CXCR} 2^{+} \mathrm{CD} 68^{+}$macrophages by blocking the CXCL8-CXCR2 axis. Combined with IFN- $\gamma$ treatment, PD1 checkpoint blockade showed significant antitumor effect. This study shows that IFN- $\gamma$ is a rational agent for improving the potency of PD1 checkpoint blockade, which could prevent TAM-mediated immune suppression in cancer therapy.

\section{METHODS}

\section{Tumor cell lines and mice}

Human PC cell lines BxPC-3, Panc-1, Miapaca-2 and healthy pancreatic cell line HPDE6-C7 were obtained from Cancer Institute of Chinese Academy of Sciences (Beijing, China). All cell lines were maintained in an appropriate cell culture medium (see online supplementary additional file 2: table S1), supplemented with $10 \%$ fetal bovine serum (HyClone, Logan, USA) and $100 \mu \mathrm{g} /$ $\mathrm{mL}$ each of penicillin and streptomycin (Gino, Hangzhou, China) in $5 \% \mathrm{CO}_{2}$ at $37^{\circ} \mathrm{C}$. All cell lines were used within 6 months of thawing. The animal experiment abided by national guidelines for the care and use of animals and approved by the ethics committee of Huzhou Central Hospital (HzhcLS2018-0807). C57BL/6 (B6) mice were purchased from Experimental Animal Center of Weitonglihua Co., Ltd. (Beijing, China), and were maintained in laminar airflow cabinets under specific pathogen-free conditions, and provided free access to irradiated pellet food and sterilized water. An ectopic inoculation method was used to establish a tumor xenograft model (six per group). Anti-PD1 blocking antibody (BioXcell Life Sciences RMP1-14, West Lebanon, USA) was used at a dose of $200 \mathrm{mg}$ per mouse and performed intraperitoneally on the indicated days. Recombinant human IFN- $\gamma$ (Wanbang Biopharma, Shanghai, China) was diluted in $2.5 \mathrm{~mL}$ of PBS $\left(4 \times 10^{5} \mathrm{U} /\right.$ bottle $)$ and given intraperitoneally with $25 \mu \mathrm{L}\left(1 \times 10^{4} \mathrm{U}\right)$ per mouse on continuous 5 days per week. Tumor tissues were harvested on indicated day after tumor implantation (day 21 after tumor implantation). Tumor volumes and survival time of each mouse was documented in the duration of the experiments. Tumor volumes were calculated using the formula: volume=length $\times$ width ${ }^{2} / 2$. The endpoint for tumor growth and survival analysis was day 42 after tumor implantation.

\section{Antibodies and flow cytometry}

Rat anti-mouse CD4 (clone GK1.5), rabbit anti-mouse CD68 (clone 2449C) and rabbit anti-mouse CXCR1 (clone 1122A) antibodies were purchased from R\&D Systems Inc. Rat anti-mouse CD8 antibody (clone 53-6.7) was purchased from BD Pharmingen. Mouse anti-human CD68 (clone Y1/82A) and mouse anti-human PD-L1 (clone MIH1) antibodies were purchased from eBioscience Inc. Mouse anti-human CXCL8 (clone E8N1), rat anti-mouse CXCR2 (clone SA045E1) and mouse antihuman CXCR2 (clone 5E8/CXCR2) antibodies were purchased from BioLegend. All antibodies were used according to the manufacturer's instructions. Intracellular cytokine staining was performed after the whole cells incubated with soluble anti-IL-2 (clone JES6-5H4; BioLegend) or anti-TNF- $\alpha$ (clone MP6-XT22; eBioscience) antibodies and monensin at $37^{\circ} \mathrm{C}$ and $5 \% \mathrm{CO}_{2}$ for 6 hours. Flow cytometry was performed with FACS Fortessa (BD Biosciences), analyzed by FlowJo V.9.0 software. In all flow cytometry assays, background versus positive staining was ascertained using isotype controls and fluorescence minus one (FMO) controls. Boxed populations quantified were identified as positive in relation to isotype and/or FMO controls.

\section{Immunostaining and immunoblotting}

Tissue sections from isolated mice tumor xenografts and human PCs were deparaffinized in xylene and rehydrated with ethanol, followed by antigen repair with EDTA buffer (PH 8.0) boiling for $10 \mathrm{~min}$, then samples were incubated with 2\% BSA for $30 \mathrm{~min}$ and added primary antibody at $4^{\circ} \mathrm{C}$ overnight. After three times PBS washes, HRP-conjugated secondary antibody (corresponding to species of primary antibody) was added for $30 \mathrm{~min}$. Following three times PBS washes, fluorophore was added for $10 \mathrm{~min}$. The fluorophores used for dualimmunofluorescence assays were FITC for CD68 and CY3 for CXCR1/CXCR2 (figure 1C), while the fluorophores 
A

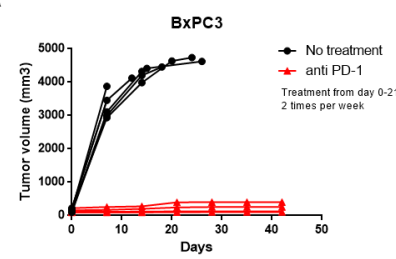

C

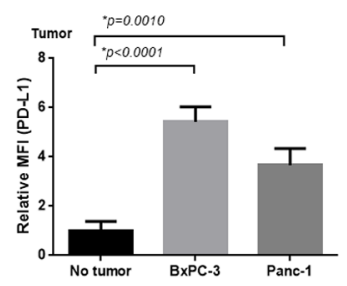

E

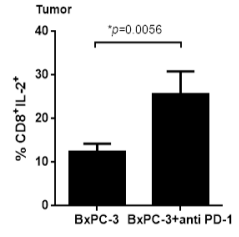

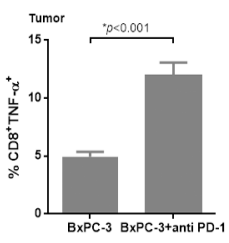

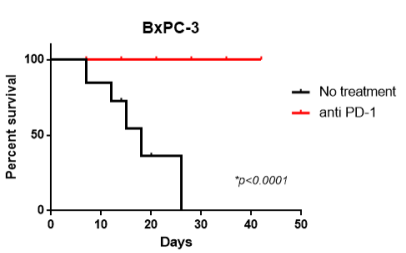

B

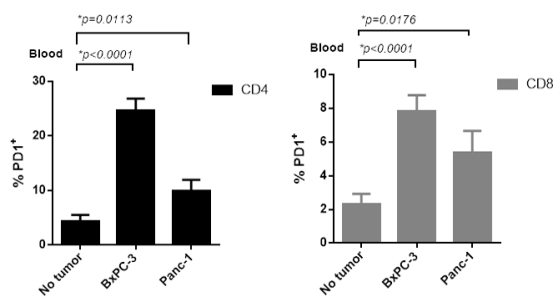

D
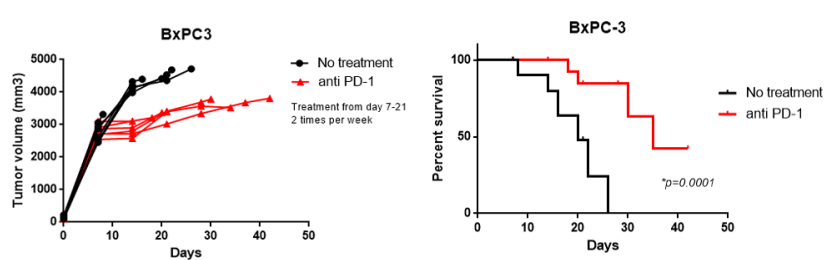

$\mathrm{F}$
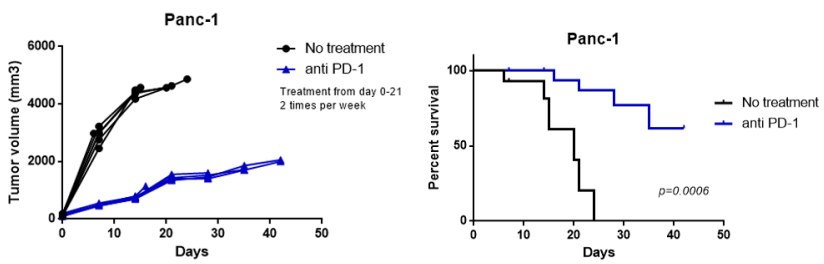

G
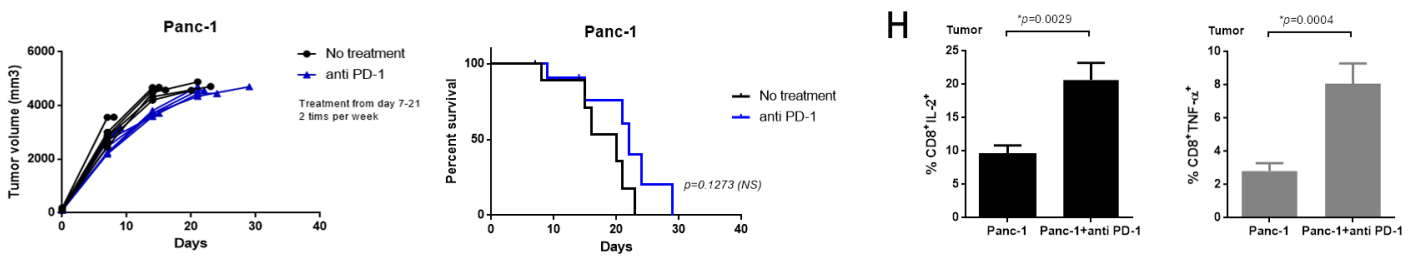

Figure 1 Effect of anti-PD1 therapy to murine pancreatic cancer (PC) tumor. Mice were ectopic inoculated with BxPC-3 and Panc-1 PC cells, control immunoglobulin G (No treatment) or anti-PD1 blocking antibody were administered intraperitoneally (200 mg per mouse, two times per week). (A) Survival and tumor volumes were monitored when anti-PD1 treatment starting on day 0 after BxPC-3 PC cells inoculation. (B) PD1 expression on circulating $\mathrm{CD}^{+}{ }^{+}$and $\mathrm{CD} 8^{+} \mathrm{T}$ cells in BxPC-3 and Panc- 1 tumorbearing mice on day 14 after tumor inoculation. (C) Surface expression of PD-L1 on BxPC-3 and Panc-1 tumor xenograft cells. (D) Survival and tumor volumes were monitored when anti-PD1 treatment starting on day 7 after BxPC-3 PC cells inoculation. $(\mathrm{E}$ and $\mathrm{H})$ Intracellular production of IL-2 and TNF- $\alpha$ on tumor-infiltrating $\mathrm{CD}^{+}{ }^{+} \mathrm{T}$ cells from BXPC-3 (E) and Panc-1 (Hh) tumorbearing mice on day 21 after delayed anti-PD1 treatment. F and G Survival and tumor volume were monitored when anti-PD1 treatment starting on day $0(\mathrm{~F})$ or on day $7(\mathrm{G})$ after Panc-1 PC cells inoculation. Experiments consist of six mice per group and are representative of at least three separate experiments. The three-dimensional data of mice tumors were measured by somatometry method. Tumor volumes were calculated using the formula: volume=length $\times$ width $^{2} / 2$. The endpoint for tumor growth and survival analysis is day 42 after tumor implantation. Two-tailed unpaired t-test or Kaplan-Meier survival analysis was used to calculate $p$ values. NSs, not significant.

used for three-marker immunofluorescence assays were CY3 for CD68, FITC for CXCR2 and CY5 for CXCL8 (figure 2). After DAPI staining for $10 \mathrm{~min}$, immunofluorescence of tissue sections was evaluated on inverted fluorescent microscope (BX-51, TR32000 Olympus, Japan) and analyzed by Image J 2X software (Rawak Software, Germany). Immunoblotting for detection of CXCL8 expression in cells and tumor tissues was performed as previously described. ${ }^{24}$ The primary antibodies used for immunoblotting were mouse anti-human CXCL8 (1:1000; BioLegend) and mouse anti-human GAPDH (1:1000, clone 1E6D9; ProteinTech). PE-conjugated goat antimouse IgG (1:2000; Santa Cruz) was used as secondary antibody. Reactive bands were detected using enhanced chemiluminescence (ECL; Amersham Bioscience, USA) immunoblotting detection reagent and analyzed by Image Lab V.5.2 software (Bioscience, USA).

\section{Real-time fluorescence quantitative PCR and ELISA}

Total RNA was extracted from cultured cells, sorted $\mathrm{CXCR}^{+} / \mathrm{CXCR}^{+}$macrophages and tumor xenografts from mice using the RNeasy Mini Kit (Qiagen, Hilden, Germany). Complementary DNA was synthesized using M-MLV reverse transcriptase (Takara Bio, Dalian, China). Real-time fluorescence quantitative PCR (RTFQ-PCR) was performed with specific primers for detecting murine arginase-1, iNOS, PD-L1, GAPDH and human CXCL8, GAPDH (see online supplementary additional file 2: table S2). The primers were obtained from Wuhan Servicebio Technology Co., Ltd., and the assays were performed 

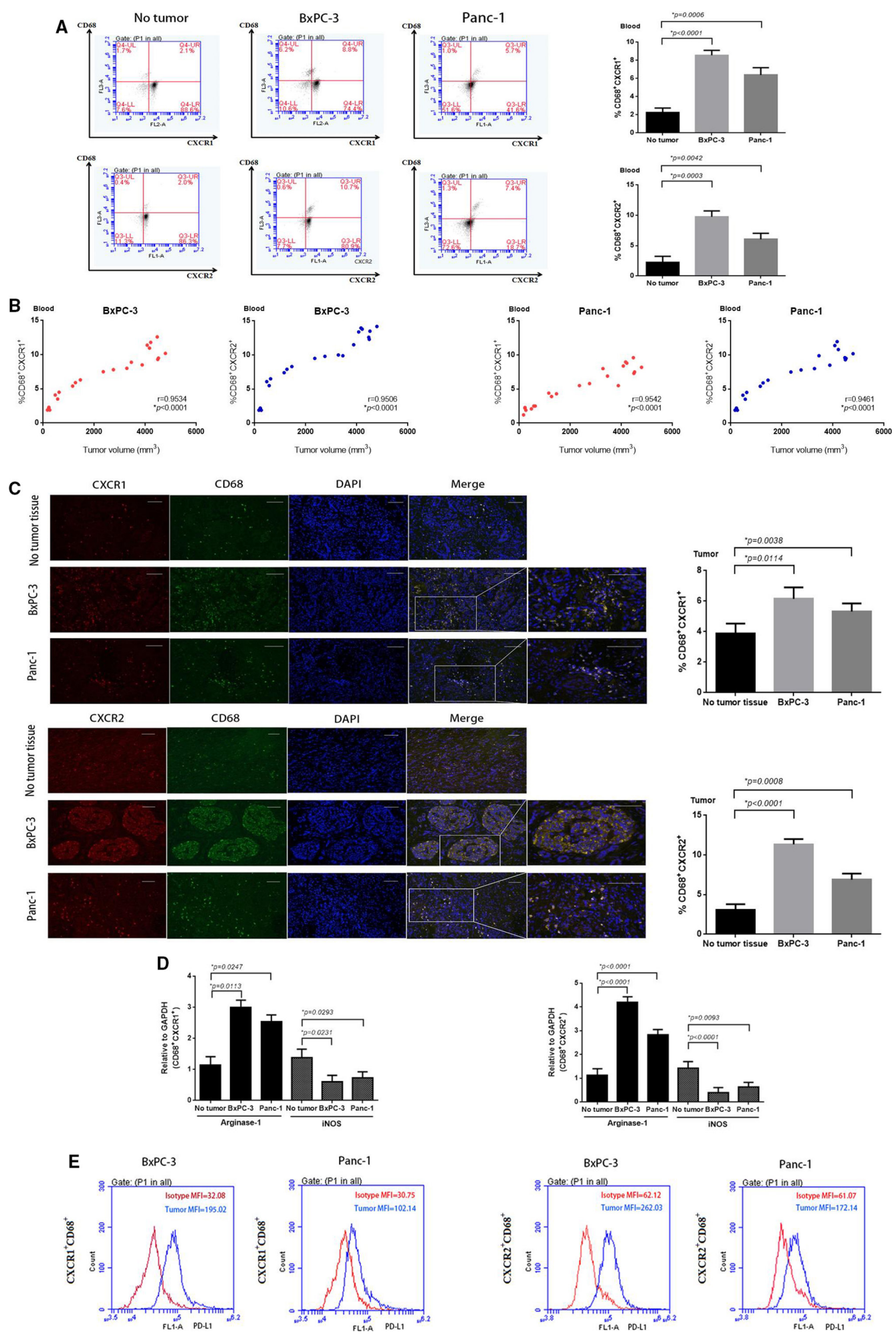

Figure 2 CXCR $1 / 2^{+} \mathrm{CD} 68^{+}$macrophages expand in murine $\mathrm{PC}$ model and associated with tumor progression. (A)

Representative fluorescence-activated cell sorting (FACS) plots and percentages of CXCR1/2+ $\mathrm{CD} 68^{+}$macrophages from mice blood on day 14 after BxPC-3 and Panc-1 PC cells inoculation. (B) Relationship between tumor volumes and the percentages of CXCR1/2+ $\mathrm{CD}^{+} 8^{+}$macrophages from tumor-bearing mice blood. (C) Tumor tissue sections immunofluorescence staining images and percentages of tumor-infiltrating CXCR1/2 ${ }^{+} \mathrm{CD} 68^{+}$macrophages from BxPC-3 and Panc-1 tumor xenografts (day 14). Scale bars represent $100 \mu \mathrm{m}$. (D) Relative quantification of arginase-1 and iNOS transcripts in CXCR1 $1^{+} \mathrm{CD} 68^{+}$and $\mathrm{CXCR} 2^{+} \mathrm{CD} 68^{+}$ macrophages sorted from BxPC-3 and Panc-1 tumor xenografts (day 14) versus circulating macrophages of non-tumorbearing mice. (E) FACS histograms showing surface expression of PD-L1 on gated populations of circulating CXCR1/2 ${ }^{+} \mathrm{CD} 68^{+}$ macrophages from BxPC-3 and Panc-1 tumor-bearing mice blood (day 14). MFI, mean fluorescent intensity. Experiments consist of five mice per group and data are representative of at least two independent experiments. Two-tailed unpaired t-test or Pearson $r$ correlation analysis was used to calculate $p$ values. 
according to the manufacturer's protocol. The PCR reactions were performed in a Bio-Rad Hard Shell 96-well microplate on a Bio-Rad CFX96 Real-Time System C1000 Touch Thermal Cycler using iTaq Universal SYBR Green Supermix (Bio-Rad) and amplified for 40 cycles. CXCL8 levels of serum samples and cell culture supernatants were detected by using the human CXCL8 sandwich ELISA kits (Dakewe Biotech, Shenzhen, China) according to the manufacturer's instructions. Normal ranges for human CXCL8 were defined by the range of values spanning (means \pm 2 SDs) obtained from the CXCL8 level data of serum from 12 healthy donors.

\section{In vitro migration assay}

Peripheral blood CXCR $1^{+} / \mathrm{CXCR}^{+}$macrophages $\left(1 \times 10^{4}\right)$ which electronically sorted from tumor-bearing mice were placed in the top chamber of a Transwell (Millipore, USA). BxPC-3 or Panc-1 cells were plated in the bottom chamber. Blocking antibodies for CXCL8, CXCR1 and CXCR2 $(50 \mu \mathrm{g} /$ well $)$ were added at the beginning of the experiment. The total number of macrophages migrating from the top to the bottom chamber after 12 hours was calculated. The migrated cells counting method was performed as previously described. ${ }^{24}$

\section{Patient samples}

Serum samples $(\mathrm{n}=44)$ from patients with $\mathrm{PC}$ were obtained at the time of hospitalization in Huzhou Central Hospital from May 2016 to January 2019, following informed consent. In these patients, 36 patients underwent radical resection and the surgical resection specimens $(n=36)$ were collected during the operation. All fresh specimens containing pancreatic tumor or adjacent normal pancreatic tissue were selected for further study. There are 25 men and 19 women in these patients (male:female $=1.32: 1$ ); the median age was 52 years (range, 37-84 years). Serum samples from healthy donors $(n=12)$ were obtained after informed consent and following approved protocols (ChiCTR1800017665).

\section{Statistical analysis}

SPSS V.19.0 was used for clinical data statistical analysis. The $\chi^{2}$ test and Fisher's exact test were used for quantitative data (table 1). Graphs for experimental data were created using GraphPad Prism software. Data in graphs were expressed as mean $\pm \mathrm{SD}$. P values were calculated in each respective figure using paired or unpaired t-test for intergroup comparison and log-rank test for survival analysis. P value $<0.05$ was considered statistically significant.

\section{RESULTS}

\section{PD1 blockade therapy shows diminished effectiveness in} established PC

First, we analyzed the effect of PD1 blockade therapy in PC xenografts using BxPC-3 cells. BxPC-3 tumors grow rapidly after tumor inoculation into the axilla of mice, contributing to $100 \%$ mortality by day 27 (figure 3A
Table 1 Correlation of CXCR2 expression on peripheral $\mathrm{CD}^{+} 8^{+}$macrophages with clinicopathological parameters in patients with pancreatic cancer

\begin{tabular}{|c|c|c|c|c|c|}
\hline \multirow[b]{2}{*}{$\begin{array}{l}\text { Clinicopathological } \\
\text { parameters }\end{array}$} & \multirow[b]{2}{*}{$\mathbf{N}$} & \multicolumn{2}{|c|}{$\begin{array}{l}\text { CXCR2 } \\
\text { expression }\end{array}$} & \multirow[b]{2}{*}{$\chi^{2}$} & \multirow[b]{2}{*}{$P$ value } \\
\hline & & $\begin{array}{l}\text { Low } \\
(n=26)\end{array}$ & $\begin{array}{l}\text { High } \\
(n=18)\end{array}$ & & \\
\hline \multicolumn{6}{|l|}{ Age (years) } \\
\hline$<65$ & 21 & 12 & 9 & 0.063 & 0.802 \\
\hline$\geq 65$ & 23 & 14 & 9 & & \\
\hline \multicolumn{6}{|l|}{ Gender } \\
\hline Male & 25 & 15 & 10 & 0.020 & 0.888 \\
\hline Female & 19 & 11 & 8 & & \\
\hline \multicolumn{6}{|l|}{ Tumor size $(\mathrm{cm})$} \\
\hline$\leq 3.0$ & 24 & 16 & 8 & 1.254 & 0.263 \\
\hline$>3.0$ & 20 & 10 & 10 & & \\
\hline \multicolumn{6}{|l|}{ Tumor site } \\
\hline Head & 33 & 20 & 13 & 0.125 & 0.723 \\
\hline Body+tail & 11 & 6 & 5 & & \\
\hline \multicolumn{6}{|l|}{ Histological grade } \\
\hline Poor+moderate & 38 & 23 & 15 & 0.238 & 0.626 \\
\hline Well & 6 & 3 & 3 & & \\
\hline \multicolumn{6}{|l|}{ Tumor classification } \\
\hline $\mathrm{T} 1-\mathrm{T} 2$ & 20 & 16 & 4 & 6.631 & $0.010^{*}$ \\
\hline T3-T4 & 24 & 10 & 14 & & \\
\hline \multicolumn{6}{|l|}{ Node classification } \\
\hline No & 18 & 14 & 4 & 4.400 & $0.036^{*}$ \\
\hline $\mathrm{N} 1+\mathrm{N} 2$ & 26 & 12 & 14 & & \\
\hline \multicolumn{6}{|l|}{ Distant metastasis } \\
\hline MO & 36 & 21 & 15 & 0.047 & 0.828 \\
\hline M1 & 8 & 5 & 3 & & \\
\hline \multicolumn{6}{|l|}{ Clinical stage } \\
\hline$|-| \mid$ & 16 & 13 & 3 & 5.107 & $0.024^{*}$ \\
\hline III-IV & 28 & 13 & 15 & & \\
\hline
\end{tabular}

and online supplementary additional file 1: figure S1a). BxPC-3 tumors constitutively overexpressed PD-L1 (figure 3C and online supplementary additional file 1: figure S2, left panel) and enhanced PD1 expression on circulating $\mathrm{CD} 4^{+}$or $\mathrm{CD}^{+} \mathrm{T}$ cells by day 14 (figure 3B and online supplementary additional file 1: figure S3a), leading us to hypothesize that the PC-induced PD1/ PD-L1 signal pathway on $\mathrm{T}$ cells contributes to immune escape in BxPC-3 tumors. Subsequently, we administered anti-PD1 blocking monoclonal antibodies (mAbs) to mice on the day of tumor inoculation and the mice showed $100 \%$ survival without evidence of tumor development (figure 3A). This indicated a role for PD1/PD-L1 signaling in immune escape by PC. To detect whether anti-PD1 treatment could lead to the regression of established BxPC-3 tumors, anti-PD1 mAbs were administered 7 days after tumor inoculation, at which time the PC tumors were well established. We found the delayed 

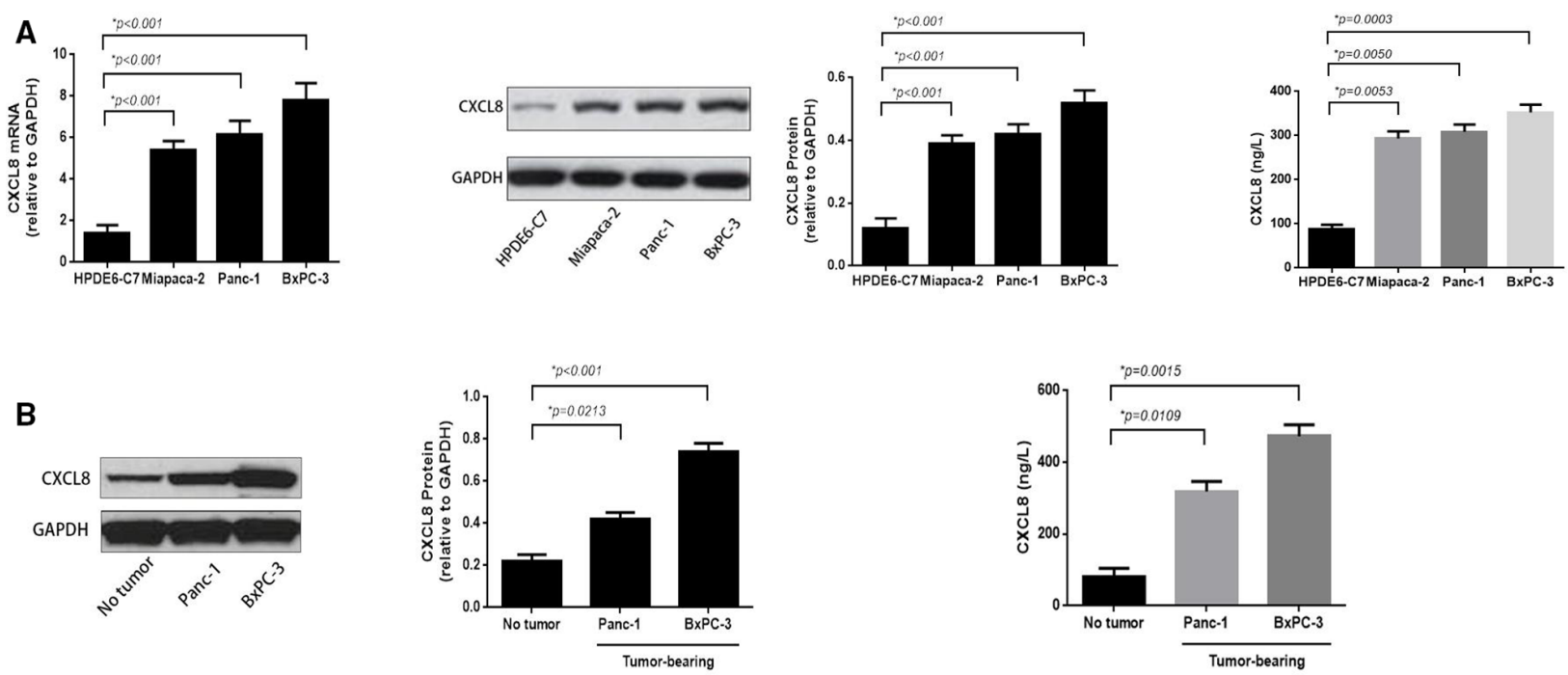

C

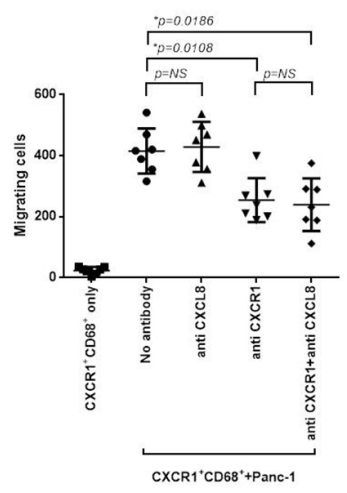

E

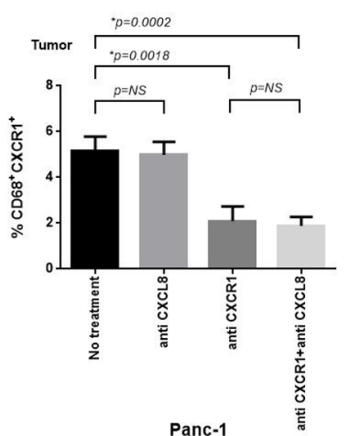

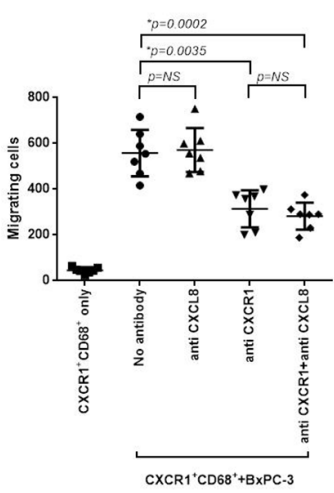

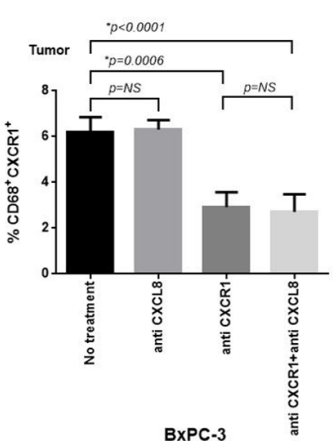

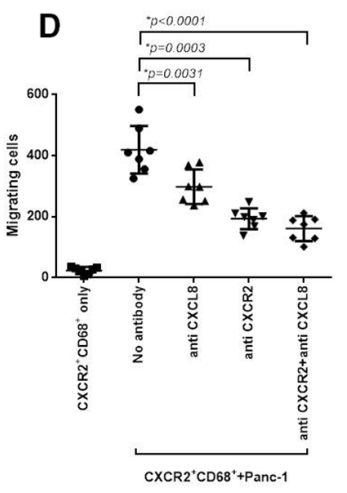

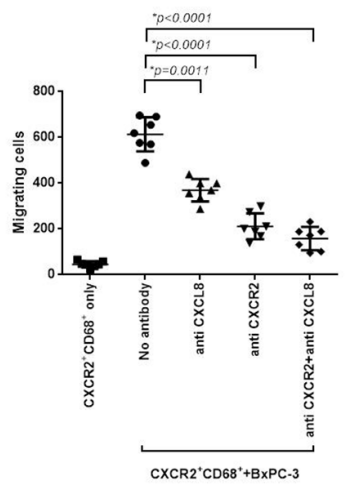

$\mathbf{F}$

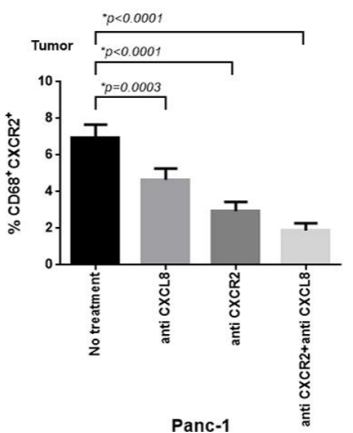

Figure 3 Murine pancreatic cancer (PC) tumor overexpresses CXCL8 and which predominantly induces $\mathrm{CXCR}^{+} \mathrm{CD}^{+} 8^{+}$ macrophages tumor trafficking. (A) RT-PCR was performed to assess the expression of CXCL8 mRNA in BXPC-3, Panc-1, Miapaca-2 PC cell lines and normal pancreatic cell line HPDE6-C7 (left panel); protein analysis (immunoblotting) for CXCL8 protein expression (middle panel); and ELISA analysis for secretion of CXCL8 in cell culture supernatants (right panel). (B) Immunoblotting was performed to detect the expression of CXCL8 protein in murine BxPC-3 and Panc-1 tumor, or adjacent non-tumor tissue (left panel); ELISA for CXCL8 detection in serum obtained from blood samples taken from BxPC3 and Panc-1 tumor-bearing mice or nomal mice (right panel). (C) In vitro migration assay was performed to calculate the absolute number of $\mathrm{CXCR} 1^{+} \mathrm{CD} 68^{+}$macrophages which electronically sorted from blood of BXPC-3 or Panc- 1 tumor-bearing mice migrating to corresponding PC cells (12 hours) when non-antibody or blocking antibodies for CXCL8, CXCR1 and CXCL8+CXCR1 $(5 \mathrm{mg} / \mathrm{mL})$ were added at the beginning of the experiment. (D) The in vitro migration assay was performed as in (C) and the migrated CXCR2 ${ }^{+} \mathrm{CD}_{68}{ }^{+}$macrophages were enumerated whereas blocking antibodies for CXCL8, CXCR2 and CXCL8+CXCR2 $(5 \mathrm{mg} / \mathrm{mL})$ were added. (E) Tumor tissue sections immunofluorescence staining was performed to calculate the tumor-infiltrating CXCR1 $1^{+} \mathrm{CD}_{68}{ }^{+}$macrophages from BXPC-3 and Panc-1 tumor when blocking antibodies for CXCL8, CXCR1 and CXCL8+CXCR1 (10 mg/per mouse) were used in tumor-bearing mice for 6 hours. (F) Immunofluorescence staining was performed as in (E) and tumor-infiltrating $\mathrm{CXCR} 2^{+} \mathrm{CD} 68^{+}$macrophages were enumerated when blocking antibodies for CXCL8, CXCR2 and CXCL8+CXCR2 (10 mg/per mouse) were used. Two-tailed unpaired t-test was used to calculate $p$ values. 
treatment modestly decreased the tumor growth and lead to a minor statistically significant improvement in survival (figure 3D). Although delayed anti-PD1 treatment showed minor therapeutic efficacy on tumor regression, BxPC-3 tumor-bearing mice after delayed anti-PD1 treatment had significantly more interleukin-2-positive $\left(\mathrm{IL}-2^{+}\right)$and tumor necrosis factor- $\alpha$-positive $\left(\mathrm{TNF}-\alpha^{+}\right) \mathrm{CD}^{+} \mathrm{T}$ cells infiltrating the tumors than untreated control mice (figure $3 \mathrm{E}$ and online supplementary additional file 1: figure S4a), suggesting that delayed anti-PD1 treatment could induce immune activation of tumor-bearing mice but it was insufficient to mediate tumor regression.

We also detected the antitumor effects of PD-1 blockade in mice inoculated with Panc-1, another PC cell line which overexpresses PD-L1 in tumor xenografts (figure 3C and online supplementary additional file 1: figure S2, right panel) and demonstrated upregulated PD1 expression on peripheral $\mathrm{T}$ cells (figure 3B and online supplementary additional file 1: figure S3b). The therapeutic efficacy of anti-PD1 treatment was similar to that observed in mice with BxPC-3 tumors when PD-1 blockade therapy was initiated on the day of tumor inoculation (figure $3 \mathrm{~F}$ and online supplementary additional file 1: figure S5a). The delayed treatment initiated on day 7 had no significant impact on tumor growth and survival rate (figure $3 \mathrm{G}$ and online supplementary additional file 1: figure S5b); however, immune activation was observed (figure $3 \mathrm{H}$ and online supplementary additional file 1: figure $S 4 b$ ).

\section{Expansion of immunosuppressive CXCR1/2 ${ }^{+} \mathrm{CD}^{+} 8^{+}$ macrophages contributes to $\mathrm{PC}$ progression}

To explore the underlying mechanisms by which PC may limit the efficacy of immune-based therapy, we analyzed TAMs in the tumors and peripheral blood of tumorbearing mice. We found that $\mathrm{CXCR} 1 / 2^{+} \mathrm{CD} 68^{+}$macrophages were increased in the peripheral blood of mice as early as day 7 after tumor inoculation (figure 1A). The proportion of $\mathrm{CXCR} 1 / 2^{+} \mathrm{CD} 68^{+}$macrophages in peripheral blood increased following the growth of BxPC-3 and Panc-1 tumors (figure 1). By day 21, CXCR1 $/ 2^{+} \mathrm{CD} 68^{+}$ macrophages represented a sizeable fraction of the monocytes in BxPC-3 and Panc-1 tumors compared with healthy tissue (figure 1C). The expansion of $\mathrm{CXCR} 1 / 2^{+} \mathrm{CD} 68^{+}$ macrophages in Panc-1 tumors was less significant than in BxPC-3 tumors, and the expansion of CXCR2 ${ }^{+} \mathrm{CD} 68^{+}$ macrophages greater than $\mathrm{CXCR} 1^{+} \mathrm{CD} 68^{+}$macrophages in PC.

To investigate whether the $\mathrm{CXCR} 1 / 2^{+} \mathrm{CD} 68^{+}$macrophages expanded in $\mathrm{PC}$ tumor-bearing mice were suppressive cells, we sorted CXCR $1 / 2^{+} \mathrm{CD} 68^{+}$cells from tumor-bearing mice and evaluated their ability to produce arginase-1 and inducible nitric oxide synthase (iNOS), mediators of macrophages immune suppression. We found that higher levels of arginase- 1 were observed in tumor-infiltrating CXCR $1 / 2^{+} \mathrm{CD} 68^{+}$macrophages which from BxPC-3 and Panc-1 tumor-bearing mice, whereas the levels of iNOS were decreased (figure 1D), suggested M2 polarization. Both circulating and tumor-infiltrating
CXCR1 $/ 2^{+} \mathrm{CD} 68^{+}$macrophages from BxPC-3 and Panc-1 tumor-bearing mice expressed higher levels of PD-L1 compared with macrophages from healthy mice (figure $1 \mathrm{E}$ and online supplementary additional file 1: figure S6). We also detected the fraction of tumor-infiltrating T-cell subsets, such as Th1 $\left(\mathrm{CD}^{+} \mathrm{IL}_{-2}{ }^{+}, \mathrm{CD} 4^{+} \mathrm{TNF}-\alpha^{+}\right)$, Th2 $\left(\mathrm{CD}^{+} \mathrm{IL}^{-} 4^{+}, \mathrm{CD}^{+} \mathrm{IL}^{-10^{+}}\right)$, Treg $\left(\mathrm{CD} 4^{+} \mathrm{FoxP}^{+}\right), \mathrm{CD} 8^{+} \mathrm{IL}^{-} 2^{+}$ and $\mathrm{CD} 8^{+} \mathrm{TNF}-\alpha^{+}$cells. We observed a significant reduction of Th1, CD8 ${ }^{+} \mathrm{IL}_{-} 2^{+}$or $\mathrm{CD} 8^{+} \mathrm{TNF}-\alpha^{+}$cells, whereas we observed an increase of Th2 or Treg cells in the tumor bed (see online supplementary additional file 1: figure S7).

\section{CXCL8 overexpressed in PC cell lines and murine PC mediates tumor trafficking of $\mathrm{CXCR2}^{+} \mathrm{CD}^{+} 8^{+}$macrophages}

We next investigated the mechanisms by which TAMs traffic to the PC tumor bed to mediate their immunosuppressive effect. We detected whether PCs produce and secrete ligands for CXCR1/2 and observed that BxPC-3, Panc-1 and Miapaca-2 PC cell lines overexpressed CXCL8 mRNA and protein, and the culture supernatant taken from these cell lines contained high concentrations of CXCL8 protein compared with a healthy pancreatic cell line HPDE6-C7 (figure 4A). In tumor-bearing mice, BxPC-3 and Panc-1 tumors overexpressed CXCL8 protein and high concentrations of CXCL8 protein was also detected in the serum (figure $4 \mathrm{~B}$ ).

To confirm whether CXCL8 induced a chemokine gradient used for CXCR1 $/ 2^{+} \mathrm{CD} 68^{+}$macrophages trafficking to the tumor bed, we used an in vitro migration assay where BxPC-3 and Panc- 1 cells were plated in the bottom chamber, and sorted CXCR1 $/ 2^{+} \mathrm{CD} 68^{+}$cells isolated from the blood of BxPC-3 and Panc-1 tumorbearing mice were added to the top chamber of a 96-well Transwell plate. Subsequently, blocking antibodies toward CXCL8, CXCR1/2 and CXCL8+CXCR1/2 were added. After 12 hours, the migration of CXCR1 $/ 2^{+} \mathrm{CD} 68^{+}$ cells was enhanced in the presence of BxPC-3 and Panc- 1 cells $(\mathrm{p}<0.0001$ and $\mathrm{p}<0.0001$, respectively) (figure 4C,D). Using CXCL8 or CXCR2 blocking antibody significantly inhibited the migration of $\mathrm{CXCR} 2^{+} \mathrm{CD} 68^{+}$cells in the Panc- $1 \quad(\mathrm{p}=0.0031$ and $\mathrm{p}=0.0003$, respectively $)$ and BxPC-3 groups $(\mathrm{p}=0.0011$ and $\mathrm{p}<0.0001$, respectively) when used alone, and their combined effects were additive $(\mathrm{p}<0.0001$ in Panc-1 group and $\mathrm{p}<0.0001$ in BxPC-3 group, respectively) (figure $4 \mathrm{D}$ ). Furthermore, the blocking antibody for CXCR1 significantly prohibited the migration of $\mathrm{CXCR} 1^{+} \mathrm{CD} 68^{+}$cells in the Panc- 1 and BxPC-3 groups ( $\mathrm{p}=0.0108$ and $\mathrm{p}=0.0035$, respectively), and CXCL8 blockade shown no inhibitory effects, similar to untreated controls ( $\mathrm{p}=0.6437$ in Panc- 1 group and $\mathrm{p}=0.8180$ in BxPC-3 group, respectively). The blockade of CXCR1 and CXCL8 simultaneously was equivalent to anti-CXCR1 used alone $(\mathrm{p}=0.3846$ in Panc- 1 group and $\mathrm{p}=0.3819$ in BxPC-3 group, respectively) (figure 4C), demonstrating that CXCL8, as a ligand of CXCR1/2, mediates a chemokine gradient effect for CXCR2 ${ }^{+} \mathrm{CD} 68^{+}$, but not $\mathrm{CXCR} 1^{+} \mathrm{CD} 68^{+}$macrophages trafficking to the 

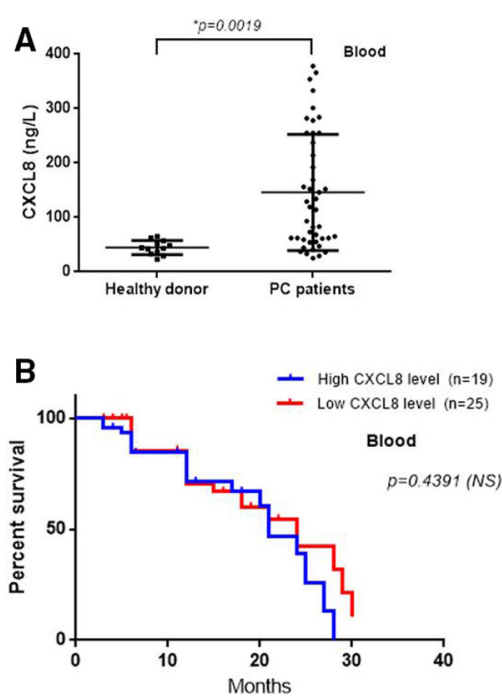
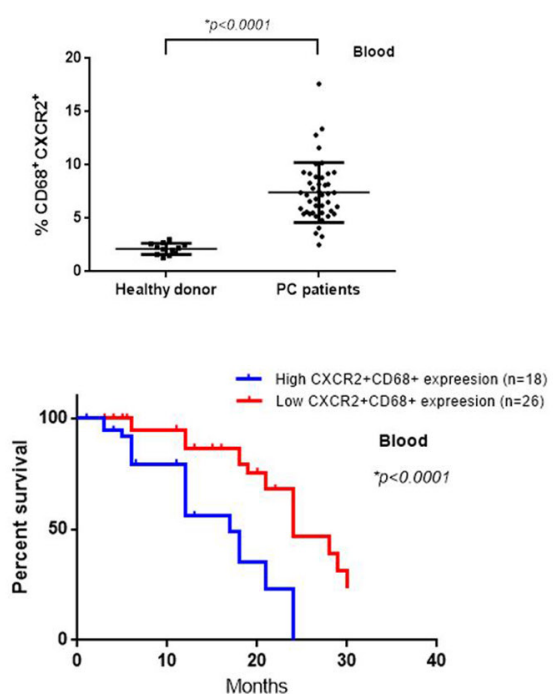
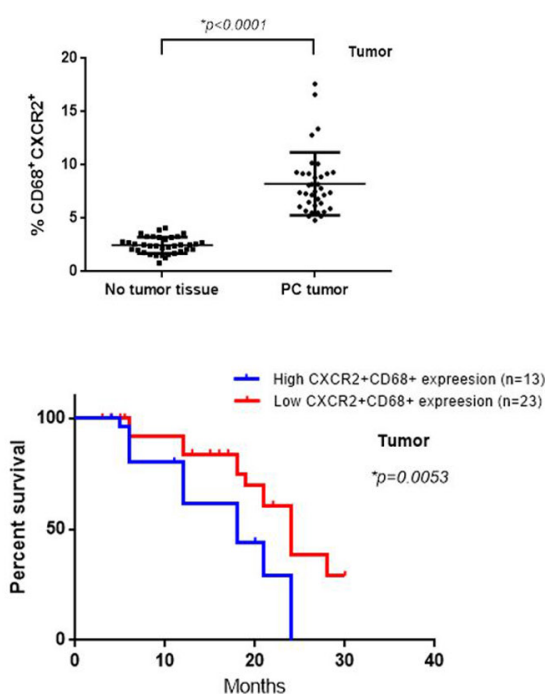

Figure 4 Human pancreatic cancer (PC) tumors secrete CXCL8. Surface expression of CXCR2 on peripheral and tumorinfiltrating $\mathrm{CD}^{+} 8^{+}$macrophages are associated with tumor progression and negatively correlate with survival in patients with PC. (A) CXCL8 were measured by ELISA in serum samples collected from healthy donors $(n=12)$ and patients with PC $(n=44)$ (left panel); flow cytometry for detection of $\mathrm{CXCR} 2^{+} \mathrm{CD} 68^{+}$macrophages percentage in peripheral blood of healthy donors $(n=12)$ and patients with PC $(n=44)$ (middle panel); tissue section immunofluorescence staining cells counting was performed in at least four random high-power fields (original magnification, $\times 400$ ) to calculate the percentage of infiltrated CXCR2 ${ }^{+} \mathrm{CD} 68^{+}$ macrophages in PC tumor tissues or adjacent non-tumor tissues $(n=36)$ (right panel). $C X C R 2^{+} C D 68^{+}$macrophages percentage was calculated as follows: $\%=\left(\mathrm{CXCR} 2^{+} \mathrm{CD} 68^{+}\right.$macrophages $/ \mathrm{CD} 68^{+}$macrophages $) \times 100$. (B) Patients with $\mathrm{PC}$ have no significant survival differences between serum CXCL8 high $(n=19)$ and low $(n=25)$ groups (left panel); whereas patients with PC with higher expression of CXCR2 on circulating (high: $n=18$, low: $n=26$; middle panel) and tumor-infiltrating (high: $n=13$, low: $n=23$; right panel) macrophages have diminished overall survival. High and low levels were defined using the median level of serum CXCL8, CXCR2 expression on peripheral or tumor-infiltrating $\mathrm{CD} 8^{+}$macrophages as the cut-off value. Two-tailed unpaired t-test or Kaplan-Meier survival analysis with log-rank test was used to calculate $p$ values. NS, not significant.

tumor bed. The same results mirrored in the in vivo experiments (figure $4 \mathrm{E}, \mathrm{F}$ and online supplementary additional file 1: figures S8 and S9).

\section{CXCL8 levels and CXCR2 expression on peripheral and tumor- infiltrating $\mathrm{CD68}^{+}$macrophages are elevated in patients with PC, which are associated with clinicopathological and prognostic significance}

Our study revealed a critical role for $\mathrm{CXCR}^{+} \mathrm{CD} 68^{+}$ macrophages in murine PC progression and CXCL8 is a key chemokine in recruiting $\mathrm{CXCR} 2^{+} \mathrm{CD} 68^{+}$macrophages to tumors. We next explored whether human PCs use the same axis. We have demonstrated that human PC cell lines secrete high concentrations of CXCL8 protein into the culture supernatants previously; thus, we measured the levels of CXCL8 in serum from patients with PC $(n=44)$ and healthy donors $(n=12)$. We found that patients with PC had higher levels of CXCL8 compared with healthy donors (patients with PC: 146.0 \pm 16.11 ; healthy donor: 44.42 $\pm 3.747, \mathrm{p}=0.0019$ ) (figure 5A, left panel). We divided patients with PC into high/low (19/25) CXCL8 level groups according to the median value of CXCL8 levels of the total patients with PC, with a follow-up of 24.3 months, the survival rate of the two groups had no significant differences (HR 1.248; 95\% CI 0.7017 to 2.414; $\mathrm{p}=0.4391$ ) (figure 5B, left panel).

We next detected CXCR2 expression on peripheral and tumor-infiltrating $\mathrm{CD}^{+} 8^{+}$macrophages and found that patients with PC have a higher percentage of CXCR $2^{+} \mathrm{CD} 68^{+}$cells in the peripheral blood compared with healthy donors $(\mathrm{p}<0.0001)$ (figure $5 \mathrm{~A}$, middle panel). Furthermore, $\mathrm{CXCR} 2^{+} \mathrm{CD} 68^{+}$macrophages represent a higher fraction in tumor tissues compared with adjacent normal pancreatic tissue $(\mathrm{p}<0.0001)$ (figure $5 \mathrm{~A}$, right panel). We divided patients with $\mathrm{PC}$ into high/low $\mathrm{CXCR}^{+} \mathrm{CD} 8^{+}$expression groups in peripheral blood $(18 / 26)$ and tumor (13/23) according to the median value respectively, and all groups were compared with clinicopathological parameters (age, sex, tumor size, tumor site, histological grade, $\mathrm{T}$ classification, $\mathrm{N}$ classification, $M$ classification, clinical stage) by $\chi^{2}$ test. We found that CXCR2 expression on peripheral $\mathrm{CD}^{+} 8^{+}$macrophages was closely correlated with $\mathrm{T}$ classification $\left(\chi^{2}=6.631\right.$, $\mathrm{p}=0.010), \mathrm{N}$ classification $\left(\chi^{2}=4.400, \mathrm{p}=0.036\right)$ and clinical stage $\left(\chi^{2}=5.107, \mathrm{p}=0.024\right)$. There was no significant correlation between CXCR2 expression and age, sex, tumor size, tumor site, histological grade and $\mathrm{M}$ classification (table 1). The same results were observed for CXCR2 expression on tumor-infiltrating $\mathrm{CD}^{+} 8^{+}$macrophages (see online supplementary additional file 2: table S3). As CXCL8 levels seemed to have no relationship with the overall survival of patients with PC, we next explored whether increased CXCR2 expression on peripheral and tumor-infiltrating $\mathrm{CD}^{+} 8^{+}$macrophages correlated with diminished overall survival in patients with PC. With a 
A

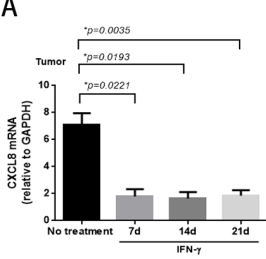

B

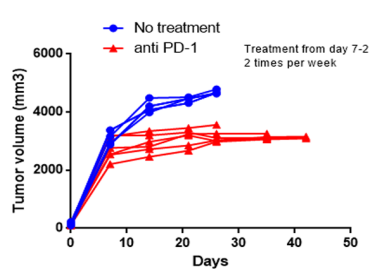

C

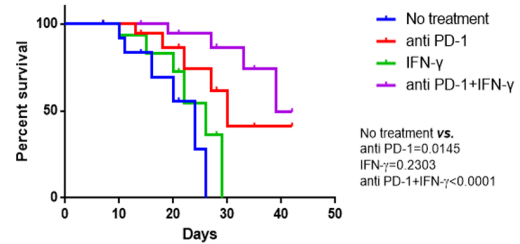

E

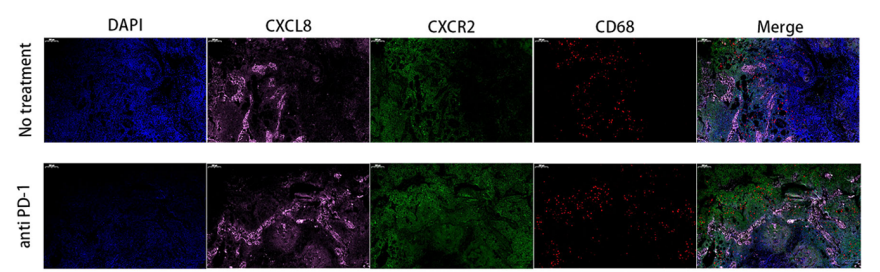

$$
\text { 章 }
$$

$\mathrm{F}$

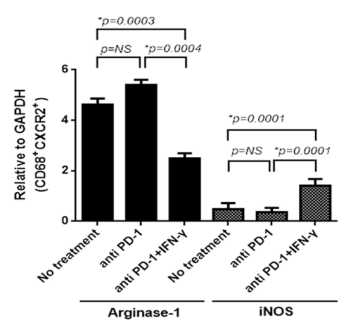

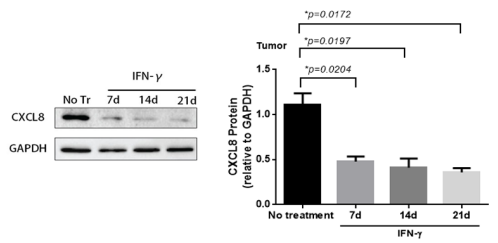
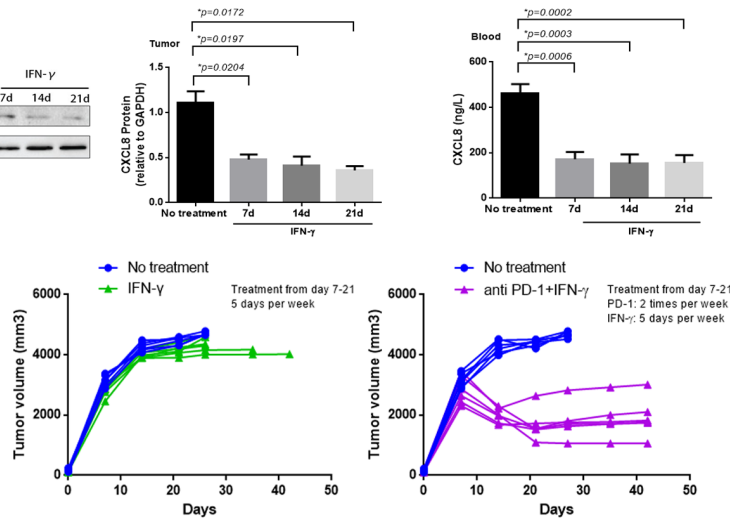

D

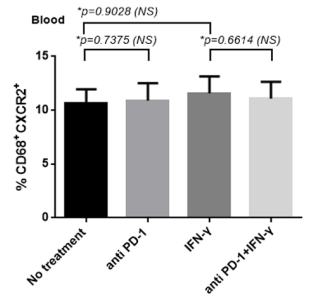

Figure 5 PD1 checkpoint blockade is more effective in tumor-bearing mice when combinatorial using IFN- $\gamma$. (A) RT-PCR was performed to assess the expression of CXCL8 mRNA in murine BxPC-3 tumors when IFN- $\gamma\left(1 \times 10^{4} \mathrm{U}\right.$ per mouse, 5 days per week) treated for 7, 14, 21 days (left panel); ilmmunoblotting assay for detection of CXCL8 protein expression after IFN- $\gamma$ treatment (middle panel); CXCL8 in serum samples collected from BxPC-3 tumor-bearing mice with IFN- $\gamma$ treatment were measured by ELISA (right panel). (B) BxPC-3 tumor volumes were monitored when anti-PD1 (200 mg per mouse, two times per week) (left panel), IFN- $\gamma\left(1 \times 10^{4} \mathrm{U}\right.$ per mouse, 5 days per week) (middle panel) or anti-PD1+IFN- $\gamma$ (right panel) treatment starting on day 7 after tumor inoculation ( $n=6$ per group). The endpoint for tumor growth analysis is day 42 after tumor inoculation. (C) Kaplan-Meier survival curves showed the overall survival of no treatment, anti-PD1, IFN- $\gamma$ or anti-PD1+IFN- $\gamma$ treatment groups and evaluated using log-rank statistics ( $n=6$ per group). The endpoint for mice survival analysis is day 42 after tumor inoculation. (D) The percentages of $\mathrm{CXCR} 2^{+} \mathrm{CD} 8^{+}$macrophages in BxPC-3 tumor-bearing mice peripheral blood from four experimental groups as in (Cc) were measured by flow cytometry (day 14). NS, not significant. (E) Tumor tissue immunofluorescence trimarkers staining images of CXCL8 and tumor-infiltrating $\mathrm{CXCR} 2^{+} \mathrm{CD} 68^{+}$macrophages from BxPC-3 tumor xenografts after anti-PD1, anti-PD1+IFN- $\gamma$ treatment or no treatment (day 21) (left panel). Scale bars represent $200 \mu \mathrm{m}$. IOD sums of CXCL8 (right top panel) and the percentages of tumor-infiltrating CXCR2 ${ }^{+} \mathrm{CD} 8^{+}$macrophages (right bottom panel) from three groups were calculated using Image-Pro Plus V.6.0 software (day 21). IOD, index of distribution. (F) RT-PCR was performed to evaluate levels of arginase- 1 and iNOS produced by sorted tumor-infiltrating $\mathrm{CXCR} 2^{+} \mathrm{CD} 68^{+}$macrophages from cohorts of mice as in (E) on day 21. NS, not significant. (G) FACS histograms showing surface expression of PD-L1 on gated populations of circulating $\mathrm{CXCR} 2^{+} \mathrm{CD}^{+} 8^{+}$macrophages from cohorts of mice as in (E) on day 21 . (Hh) RT-PCR was performed to detect the mRNA expression of PD-L1 in sorted tumor-infiltrating CXCR2 ${ }^{+} \mathrm{CD} 68^{+}$macrophages from cohorts of mice as in (E) on day 21 . MFI, mean fluorescent intensity. Two-tailed unpaired t-test or Kaplan-Meier survival analysis with log-rank test was used to calculate $p$ values. 
follow-up of 24.3 months, the median survival of patients with high $(\mathrm{n}=18)$ versus low $(\mathrm{n}=26)$ CXCR2 expression on peripheral $\mathrm{CD} 68^{+}$macrophages was 17.0 months versus 24.0 months (HR 3.358; 95\% CI 3.138 to 14.99 ; $\mathrm{p}<0.0001$ ) (figure 5B, middle panel). Furthermore, high $(n=13)$ versus low $(n=23)$ CXCR2 expression on tumor-infiltrating $\mathrm{CD}^{+} 8^{+}$macrophages showed a median survival of 18.0 months versus 24.0 months (HR 2.416; $95 \%$ CI 1.588 to $8.434 ; p=0.0053$ ) (figure $5 \mathrm{~B}$, right panel). These results demonstrated that CXCL8 is an essential chemokine for the recruitment of CXCR2 ${ }^{+} \mathrm{CD} 68^{+}$macrophages in murine PC tumors as well as in human patients with PC. Furthermore, the expansion of $\mathrm{CXCR}^{+} \mathrm{CD} 68^{+}$ macrophages contributes to human PC progression and poor prognosis.

\section{IFN- $\gamma$ suppresses the secretion of CXCL8 in PC tumors, preventing CXCR2 ${ }^{+} \mathrm{CD} 8^{+}$macrophage trafficking to the tumor microenvironment and enhancing PD1 blockade efficacy}

CXCL8 and CXCR2 ${ }^{+} \mathrm{CD} 68^{+}$macrophages are capable of promoting PC progression and limiting antitumor T-cell responses and our previous studies showed that IFN- $\gamma$ suppresses CXCL8 in BxPC-3 and Panc-1 cell lines $^{24}$ (see online supplementary additional file 1: figure S10); thus, we sought to investigate whether anti-PD1 therapy combined with using IFN- $\gamma$ was more effective at reducing tumor progression. We focused on the BxPC-3 model because PD1 blockade therapy is highly efficacious and has a significant expansion of $\mathrm{CXCR} 2^{+} \mathrm{CD} 68^{+}$ macrophages. We tested the expression and secretion of CXCL8 in IFN- $\gamma$ treated tumor-bearing mice and found that the expression of CXCL8 mRNA and protein decreased on days 7, 14 and 21 after IFN- $\gamma$ treatment. These results mirrored the concentrations of CXCL8 protein in the serum of tumor-bearing mice (figure 2A). Mice were inoculated with BxPC-3 cells on day 0, and treatment begun on day 7 , when the tumors were well established. Anti-PD1 or IFN- $\gamma$ treatment alone was ineffective at preventing tumor growth (figure 2B, left and middle panel). In contrast, mice treated with antiPD1+IFN- $\gamma$ experienced significant reductions in tumor growth (figure 2B, right panel) (see online supplementary additional file 1: figure S11). Kaplan-Meier survival analysis in BxPC-3 tumor-bearing mice showed a modest but significant difference in median survival between no treatment and the anti-PD1 groups (median survival: 24.0 days vs 30.0 days; HR 3.704; $95 \%$ CI 1.539 to 24.05 ; $\mathrm{p}=0.0145$ ), while mice receiving IFN- $\gamma$ treatment did not have an increase in the median survival (median survival: 24.0 days vs 26.0 days; HR 1.885 ; $95 \%$ CI 0.6659 to 7.179 ; $\mathrm{p}=0.2303)$. Conversely, mice treated with anti-PD1+IFN- $\gamma$ experienced a significant increase in median survival (median survival: 24.0 days vs 39.0 days; HR 8.103 ; $95 \%$ CI 8.199 to $249.2 ; \mathrm{p}<0.0001$ ) (figure $2 \mathrm{C}$ ).

We next explored whether the efficacy of combinatorial therapy was related to the downregulation of CXCL8 and reduction of CXCR2 ${ }^{+} \mathrm{CD} 68^{+}$macrophages in tumor-bearing mice. We found that the percentage of circulating $\mathrm{CXCR} 2^{+} \mathrm{CD} 68^{+}$macrophages in mice were no different between control, anti-PD1 or IFN- $\gamma$ used alone and the combined treatment groups (figure 2D). The expression of CXCL8 was modestly increased in BxPC-3 tumors after anti-PD1 treatment but was significantly decreased following anti-PD1+IFN- $\gamma$ treatment; however, tumor-infiltrating $\mathrm{CXCR}^{+} \mathrm{CD}^{+} 8^{+}$macrophages represented a minor increased fraction in tumor tissue with PD1 blockade therapy but were largely diminished after anti-PD1 +IFN- $\gamma$ treatment (figure 2E).

The $\mathrm{CXCR}^{+} \mathrm{CD} 68^{+}$macrophages expanded in tumorbearing mice were suppressive cells; thus, we further investigated whether these cells would change the immunosuppressive nature after anti-PD1+IFN- $\gamma$ treatment. To this end, we sorted $\mathrm{CXCR}^{+} \mathrm{CD} 68^{+}$macrophages from tumor-bearing mice and evaluated their capacity to produce arginase-1 and iNOS. We observed that increased, but not significant, levels of arginase-1 were detected in tumor-infiltrating $\mathrm{CXCR}^{+} \mathrm{CD} 68^{+}$macrophages after anti-PD1 treatment, which significantly decreased following anti-PD1+IFN- $\gamma$ treatment. The levels of iNOS in tumor-infiltrating $\mathrm{CXCR} 2^{+} \mathrm{CD}_{6} 8^{+}$macrophages were no different between no treatment and anti-PD1 treated mice; however, iNOS was significantly increased in these cells from anti-PD1+IFN- $\gamma$ treated tumor-bearing mice (figure $2 \mathrm{~F}$ ), suggesting these cells showed M1 polarization. We also observed higher levels of PD-L1 expression in circulating and tumor-infiltrating $\mathrm{CXCR} 2^{+} \mathrm{CD}^{+} 8^{+}$ macrophages from tumor-bearing mice after anti-PD1 treatment, whereas the expression of PD-L1 was significantly decreased in these cells following anti-PD1+IFN- $\gamma$ treatment (figure 2G,H). We also observed that the fraction of tumor-infiltrating Th1 $\left(\mathrm{CD} 4^{+} \mathrm{IL}-2^{+}, \mathrm{CD} 4^{+} \mathrm{TNF}-\alpha^{+}\right)$, $\mathrm{CD}^{+} \mathrm{IL}_{-} 2^{+}$and $\mathrm{CD} 8^{+} \mathrm{TNF}^{-} \alpha^{+}$cells minor increased after anti-PD1 or IFN- $\gamma$ treatment alone while significantly increased following anti-PD1+IFN- $\gamma$ treatment; whereas Th2 $\left(\mathrm{CD}^{+} \mathrm{IL}^{-10^{+}}\right)$and Treg $\left(\mathrm{CD} 4^{+} \mathrm{FoxP}^{+}\right)$cells minor decreased after anti-PD1 or IFN- $\gamma$ treatment alone while significantly reduced following anti-PD $1+$ IFN $-\gamma$ treatment in tumor bed (see online supplementary additional file 1: figures S12 and S13). Together, these results suggest that IFN- $\gamma$ improves the efficacy of anti-PD1 therapy. IFN- $\gamma$ could suppress the expression of tumor-derived CXCL8 to inhibit tumor-induced trafficking of $\mathrm{CXCR} 2^{+} \mathrm{CD}^{+} 8^{+}$ macrophages to the tumor bed. In addition, the peripheral and tumor-infiltrating $\mathrm{CXCR} 2^{+} \mathrm{CD} 68^{+}$macrophages alter the immunosuppressive properties, while the T-cell subsets turn to a state of immune activation after anti-PD1 combined with IFN- $\gamma$ treatment.

\section{DISCUSSION}

PD1 receptor is an important immune checkpoint molecule, which controls inflammation and self-reactivity in physiological conditions. ${ }^{4-6}$ However, cancers often use the PD1/PD-L1 signaling axis to induce the apoptosis of tumor-reactive cytotoxic T cells to mediate tumor immune escape. ${ }^{71029}$ T-cell checkpoint-based immunotherapy has 
widely been used in the treatment of patients with melanoma, non-small cell lung cancer and renal cell carcinoma with objective antitumor responses observed in 6\%-28\% of these patients. ${ }^{30}$ Although PD1 immune checkpoint blockade has shown promise in cancer therapy and induced durable responses in several tumor types, its efficacy is limited for patients with PC. ${ }^{11}$ In the present study, the murine model of PC presented here also illustrates the limitations of PD1 checkpoint blockade as a singleagent treatment. Tumor development was completely inhibited when anti-PD1 treatment began at the time of tumor inoculation but had only minor effects on established tumors. These results showed that factors beyond PD1 signaling contribute to tumor immune evasion in PC tumors. PC is characterized by a dense, fibrotic stroma that can trap tumor-infiltrating cytotoxic $\mathrm{T}$ cells in peritumoral tissues and thus can prevent them from reaching the vicinity of tumor cells. Moreover, the immunosuppressive TME within the stroma also dampens the activity of tumor-infiltrating T lymphocytes. ${ }^{3}{ }^{32} \mathrm{~A}$ minor percentage of patients with PC benefited from PD1 checkpoint inhibitors used alone, suggesting that other mechanisms also contribute to immune evasion. Combinatorial strategies may be a rational option to overcome the resistance of cancers to PD1 immune checkpoint blockade by enhancing cytotoxic T-cell tumor infiltration, rendering $\mathrm{T}$ cells resistant to PD1/PD-L1-mediated anergy or simultaneously blocking other mechanisms of tumor immune escape. ${ }^{29} 3334$ Several clinical trials demonstrated that combination regimens appear to be more potent than single agents, with objective response rates of $53 \%$ in metastatic melanoma, $30.8 \%$ in gastric or esophagogastric junction cancer and $50 \%$ in advanced hepatocellular carcinoma. ${ }^{35-37}$ Despite the progress of combinatorial treatment with PD1 blockade in melanoma or non-melanoma solid cancers, PC has proven refractory to PD1 checkpoint-based immunotherapy even when combined with chemotherapy (capecitabine, gemcitabine or nab-paclitaxel) or combined with other immunotherapy (tremelimumab: antibody targeting CTLA-4; acalabrutinib: bruton tyrosine kinase inhibitor; mogamulizumab: antibody targeting CC chemokine receptor 4; cabiralizumab: antibody targeting CSF-1 receptor). ${ }^{11}$ These pieces of clinical evidence encourage us to pay more attention to combinations of checkpoint inhibitors with other modalities that could potentially change the tumor-generated TME or target other mechanisms of immune escape.

TAMs constitute an important component of the TME. ${ }^{12}$ M2 polarized TAMs are considered immunosuppressive cells and can induce a favorable environment for cancer by inhibiting the immune system. Expansion of M2 polarized TAMs is a major mechanism used by cancers to escape immune surveillance, by upregulating the expression of checkpoint molecules, prohibiting tumor-reactive cytotoxic T-cell activation, or producing more cytokines and growth factors that directly sustain tumor cells. ${ }^{15-17}$ In PC tumor-bearing mice, we observed significant increases in the frequency of CXCR1 $/ 2^{+} \mathrm{CD} 68^{+}$macrophages in peripheral blood and tumors. CXCR $1^{+} \mathrm{CD} 68^{+}$macrophages accounted for up to $8.3 \%$ (BxPC-3) and $6.1 \%$ (Panc-1) of the total cells in the blood, $6.1 \%$ (BxPC-3) and 5.5\% (Panc-1) of the total cells in the tumor, whereas $\mathrm{CXCR} 2^{+} \mathrm{CD} 68^{+}$macrophages increased to $9.8 \%$ and $6.7 \%$ in the blood of BxPC-3 and Panc- 1 tumor-bearing mice and to $10.6 \%$ and $6.4 \%$ in the tumors of these mice, respectively. The fraction of circulating CXCR1 $/ 2^{+} \mathrm{CD} 68^{+}$macrophages positively correlates with tumor volume. These findings illustrated that tumorinduced expansion of $\mathrm{CXCR} 1 / 2^{+} \mathrm{CD}^{+} 8^{+}$macrophages is associated with $\mathrm{PC}$ progression and $\mathrm{CXCR} 2^{+} \mathrm{CD} 68^{+}$ macrophages were preferentially expanded in two types of PC cell-based tumors. Moreover, the tumor-infiltrating CXCR1 $/ 2^{+} \mathrm{CD}_{68}{ }^{+}$macrophages in our model produced higher levels of arginase-1 and decreased levels of iNOS. Arginase-1 is an amino acid-degrading enzyme which has influence on immunogenic tolerance and contributes to the functional failure of $\mathrm{T}$ cells. ${ }^{38}$ Conversely, iNOS significantly reversed $\mathrm{T}$ cells suppression, ${ }^{39}$ and this result suggested that $\mathrm{PC}$ tumor-infiltrating $\mathrm{CXCR} 1 / 2^{+} \mathrm{CD} 68^{+}$ macrophages are M2 polarized TAMs and mediate immunosuppressive effects on $\mathrm{T}$ cells. In humans with PC tumors, similar patterns were found, demonstrating that the expansion of $\mathrm{CXCR} 2^{+} \mathrm{CD} 68^{+}$macrophages also contributes to human PC progression and poor prognosis.

In humans, CXCL8 is one of the most important CXC chemokine ligands and its signal is mediated through both CXCR1 and CXCR2. ${ }^{25}$ CXCL8 expression is significantly upregulated in human colon, head and neck, pancreatic and esophageal cancers compared with healthy tissues based on previously published literature. ${ }^{25} 27$ In the present study, we also confirmed this result in a murine model of PC. As CXCL8 expression is significantly different between mice and humans (mice lack a CXCL8 homolog), so the CXCL8 detected in tumor-bearing mice was totally derived from the human PC cells. CXCR1/2-expressing TAMs are migratory hematopoietic cells and have an immunosuppressive nature (M2 polarization). They have been shown to expand in murine and human PC tumors; thus, these cells may be recruited to the tumor bed by tumor-derived CXCL8 through the CXCL8-CXCR1/2 axis to facilitate PC immune escape. In our in vitro migration assay experiment shown in figure $4 \mathrm{C}, \mathrm{D}$, we present a significant reduction in the number of $\mathrm{CXCR} 2^{+} \mathrm{CD} 68^{+}$macrophages that migrate toward a tumor attractant when we use antiCXCL8 and/or anti-CXCR2 blockade, whereas the migration of $\mathrm{CXCR} 1^{+} \mathrm{CD} 68^{+}$macrophages is not completely inhibited when CXCL8 is absent, suggesting that CXCL8 predominantly mediated the tumor trafficking of CXCR2expressing TAMs. This result also been proved by our in vivo experiments. In a review of previous published articles, Ijichi showed that CXCL8 overexpressing human PC cells mixed with pancreatic fibroblasts increased tumor growth in a mouse model. In addition, CXCR2 blockade treatment in the stromal cells delayed tumor progression 
in a mixed cells xenograft model, demonstrating the interaction between tumor-derived CXCL8 and stromal CXCR2 promotes tumor progression, invasion and metastasis. $^{28}$ Trellakis et al indicated that deletion of CXCR2 in a CXCL8 upregulated colitis-associated tumorigenesis mouse model reduced the number of granulocytic myeloid-derived suppressor cells (GrMDSCs), which mediated a marked effect on T-cell function. ${ }^{40}$ Chen $e t$ $a l$ showed expression of CXCR1 positively correlates with expansion of cancer stem cells (CSCs) in PC, exogenous CXCL8 promoted PC cells proliferation, invasion ability, tumorsphere formation and CSCs population in vitro, and CXCR1 blockade treatment reversed these effects. ${ }^{27}$ Finally, one study indicated that CXCR1 predominantly engaged mouse CXCL6, another CXC chemokine ligand, which binds to both CXCR1 and CXCR2. ${ }^{41}$ CXCR1 and CXCR2 likely both play a role in modulating tumor stromal cell trafficking to the tumor bed via CXCL8 or other CXC chemokine ligands depending on the model system studied. In our model, PC tumor-derived CXCL8 mainly mediated $\mathrm{CXCR} 2^{+} \mathrm{CD} 68^{+}$macrophage trafficking to the tumor bed.

IFN- $\gamma$ is an important activator of innate and adaptive immunity and possesses antiviral, immunoregulatory, and anti-tumor properties. It modulates transcription in up to 30 genes via an IFN- $\gamma$ signaling pathway to mediate a variety of physiological and cellular responses. ${ }^{22}$ The in vitro studies on the direct killing effect of IFN- $\gamma$ on cancer cells are extensive and the results indicate that IFN- $\gamma$ has anti-proliferative activity leading to growth inhibition or cell death by apoptosis or autophagy. ${ }^{24}{ }^{42}$ However, the evidence to support the contribution of IFN- $\gamma$ to significant tumor regression is sparse in in vivo studies and clinical trials. ${ }^{43}$ Based on its immunoregulatory characteristics, it is theorized that IFN- $\gamma$ induces the activation of the immune system in multiple ways to promote antitumor immune responses, including enhancing natural killer (NK) cell activity, antigen presentation and lysosome activity of macrophages, inducing M1 polarization and iNOS production and the production of IgG2a and IgG3 from activated plasma B cells, increasing the expression of MHC-I molecules in tumor cells, and increasing the expression of MHC-II molecules on antigen-presenting cells. ${ }^{44}$ On the other hand, there are opposing effects of IFN- $\gamma$ that attenuate immune function based on findings that IFN- $\gamma$ promotes T-cell exhaustion through upregulating the coregulatory molecules B7-H1 (PD-L1) and B7-DC (PD-L2), inducing T-cell apoptosis via the PD1/ PD-1 checkpoint signaling pathway. ${ }^{45}$ Benci et al recently showed that blocking tumor IFN- $\gamma$ signaling decreased interferon-stimulated genes (ISGs) in cancer cells, while in immune cells, ISGs were increased by enhancing IFN- $\gamma$ production in exhausted T cells (T-EXs). In nonimmunogenic tumors, T-EXs use IFN- $\gamma$ to drive the maturation of innate immune cells. Thus, IFN- $\gamma$ signaling in cancer cells and immune cells has opposing effects, establishing a regulatory relationship that limits both adaptive and innate immune killing, and the perturbation of this relationship is associated with the immune checkpoint blockade response, which is independent of the tumor mutational burden. These results presented a possible mechanism in which the opposing effects of IFN- $\gamma$ were integrated and impacted PD1 checkpoint-based immunotherapy. ${ }^{46}$ Although the pleiotropic activities of IFN- $\gamma$ mostly involve its immunostimulatory and immunomodulatory effects that enhance antitumor immune responses, accumulating evidence indicates that IFN- $\gamma$ may regulate the expression and secretion of tumor-derived cytokines and growth factors, thereby contributing to the alteration of the tumor microenvironment. ${ }^{478}$

In our previous study, we proved that IFN- $\gamma$ suppressed the expression and secretion of CXCL8 in PC cell lines and inhibited the proliferation and migration of these cells. $^{24}$ In the present study, we confirmed that IFN- $\gamma$ treatment also reduced tumor-derived CXCL8 expression in PC xenograft tumors and its secretion in mouse peripheral blood. As the CXCL8-CXCR1/2 signaling axis is essential for the pathogenesis of cancer, and the interaction between CXCL8 secreted by cancer cells and CXCR1/2 in the tumor microenvironment is critical for cancer progression and metastasis, ${ }^{25}{ }^{28}$ we next investigated the biologic ramifications of IFN- $\gamma$ combined with anti-PD1 treatment on murine PC tumors. Remarkably, combining PD1 checkpoint blockade with IFN- $\gamma$ treatment showed significantly enhanced antitumor effects and prolonged the survival time of the mice compared with anti-PD1 or IFN- $\gamma$ treatment used alone, despite systemic accumulation of $\mathrm{CXCR} 2^{+} \mathrm{CD} 68^{+}$macrophages in the peripheral blood of the mice. CXCL8 was overexpressed and $\mathrm{CXCR} 2^{+} \mathrm{CD} 68^{+}$macrophages accumulated in the mouse tumor bed when anti-PD1 monotherapy was administered, while combinatorial treatment significantly reduced the expression of CXCL8 and diminished $\mathrm{CXCR}^{+} \mathrm{CD} 8^{+}$macrophage tumor infiltration. Interestingly, the retained tumor-infiltrating $\mathrm{CXCR}^{+} \mathrm{CD} 68^{+}$ macrophages converted and produced less arginase- 1 and more iNOS and then showed an M1 polarization. These results indicated that when PD1 blockade monotherapy promotes a cytotoxic T cell-mediated tumoricidal effect, the expression of tumor-derived CXCL8 increases, thus activating the CXCL8-CXCR2 axis, enhancing CXCR2expressed M2 TAM tumor trafficking and infiltration and inducing immunosuppressive effects on $\mathrm{T}$ cells as a rebound mechanism. However, IFN- $\gamma$ reverses this opposing effect by blocking the CXCL8-CXCR2 axis by downregulating the expression of CXCL8 and inhibiting CXCR2-expressing M2 TAM tumor trafficking and infiltration, thus promoting the antitumor effects of PD1 blockade in murine PC tumors. As IFN- $\gamma$ has increasingly been regarded as a key stimulus that induces the M1 polarization of TAMs, IFN- $\gamma$ may act directly on tumorinfiltrating macrophages and then convert these cells to the M1 subtype. This may be another mechanism by which IFN- $\gamma$ enhances the efficacy of PD1 blockade therapy.

The clinical relevance of these findings is provided by studies in human patients with PC. We demonstrated that 


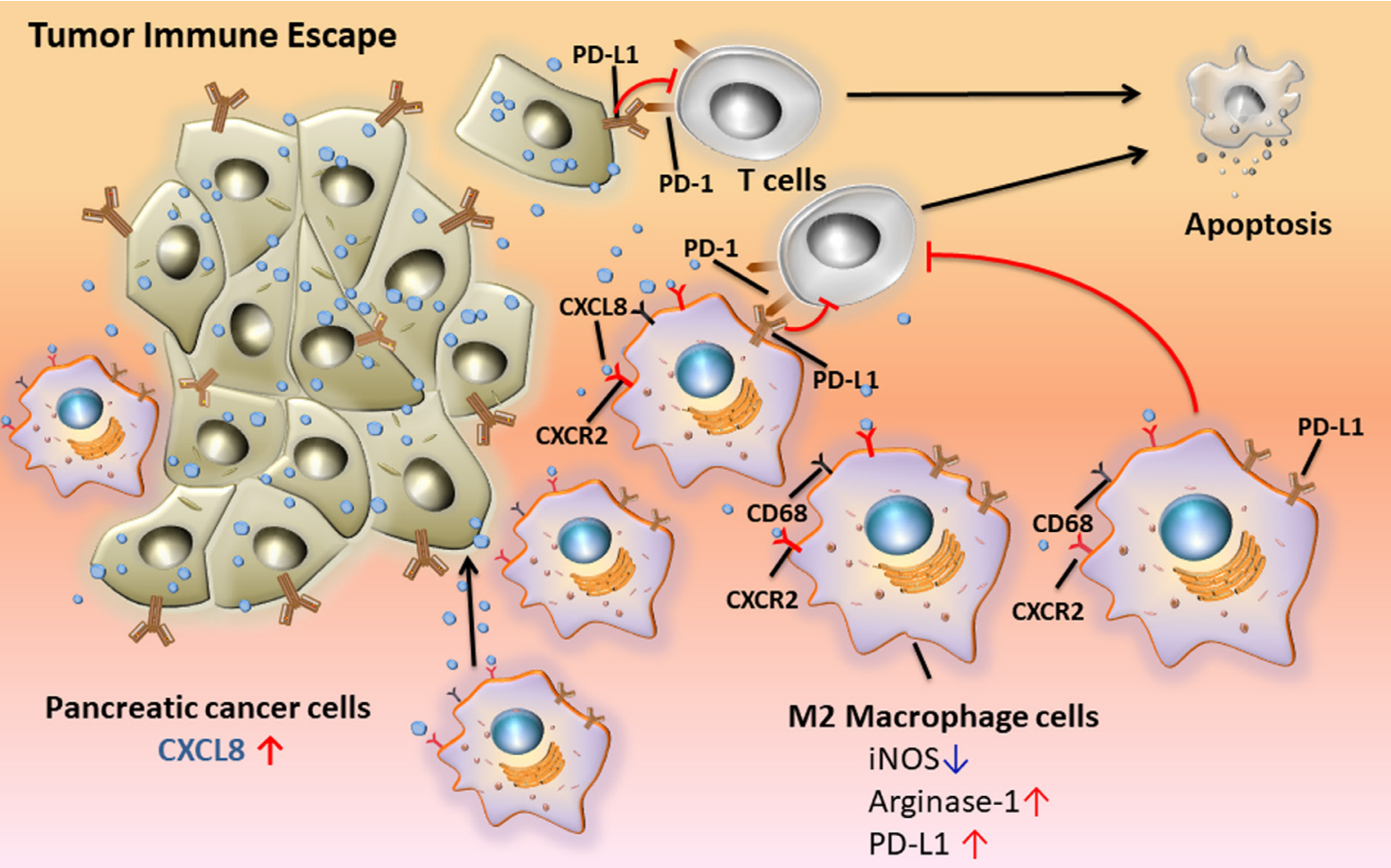

\section{Tumor Death}

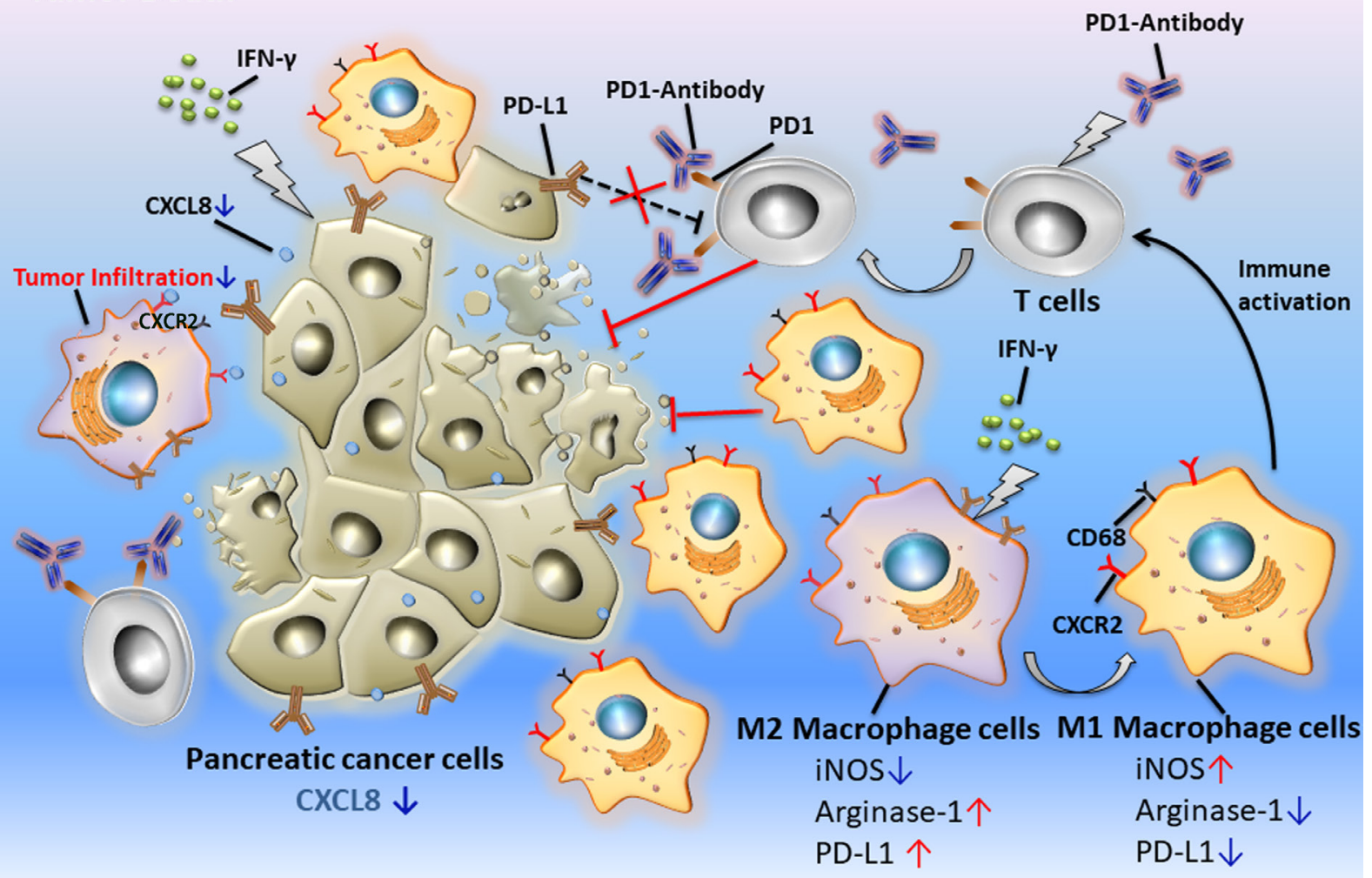

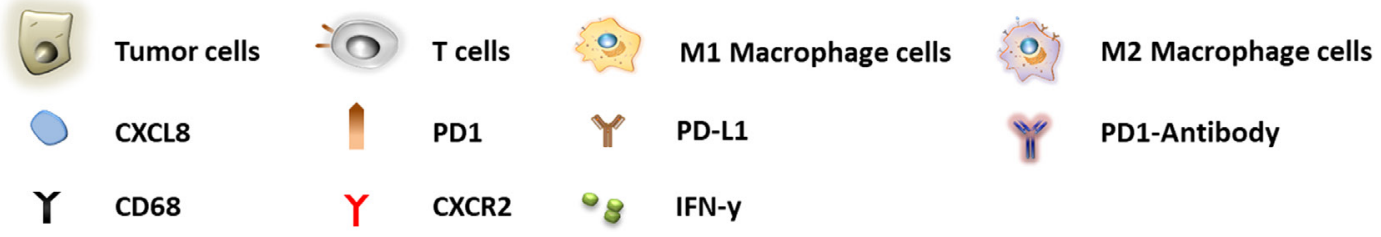

Figure 6 Graphical abstract. 
CXCL8 levels are elevated in peripheral blood of patients with PC. Meanwhile, patients with PC presented elevated CXCR2 expression on peripheral and tumor-infiltrating CD68 ${ }^{+}$macrophages, which are associated with clinicopathological and prognostic significance. These results suggest that the tumor-induced CXCL8 upregulation and $\mathrm{CXCR}^{+} \mathrm{CD} 68^{+}$macrophage expansion observed in tumor-bearing mice is also active in patients with $\mathrm{PC}$ and the expansion of $\mathrm{CXCR}^{+} \mathrm{CD} 68^{+}$macrophages also contributes to human PC progression and poor prognosis. Thus, our results showed a close correlation between our in vivo study and clinical observation.

The potential for clinical application of these findings is notable because there are currently no clinically relevant evidence to show that anti-PD1 immunotherapy is effective in treating PC. ${ }^{11}$ Conversely, IFN- $\gamma$ is also not approved for the treatment of any cancer, although studies showed improved survival when IFN- $\gamma$ was administered to patients with bladder carcinoma, malignant melanoma and advanced ovarian carcinoma. ${ }^{43}$ In the present study, we reported that IFN- $\gamma$ suppresses the expression and secretion of tumor-derived CXCL8, which inhibits tumor-infiltrating of $\mathrm{CXCR}^{+} \mathrm{CD}^{+} 8^{+}$macrophages by blocking the CXCL8-CXCR2 axis. This ultimately enhances the anti-PD1 efficacy in a murine PC model. Conversely, human PC also produces high levels of CXCL8. Patients with PC had elevated CXCR2 expression on peripheral and tumor-infiltrating $\mathrm{CD}^{+} 8^{+}$macrophages, which are associated with advanced tumor stage and poor prognosis. Our findings indicate that IFN- $\gamma$ is a translatable, therapeutic option to improve the efficacy of PD1 blockade therapy in patients with PC by preventing the tumor trafficking of $\mathrm{CXCR} 2^{+} \mathrm{CD}_{6} 8^{+}$macrophages blocking the CXCL8-CXCR2 axis.

Limitations of this work include the absent information regarding whether IFN- $\gamma$ also influences the differentiation of tumor-infiltrating T-cell subsets (such as Th1, Th2, Th17 and Treg cells) and whether the variable $\mathrm{CXCR}^{+} \mathrm{CD} 8^{+}$macrophage infiltration has associations with the tumor-infiltrating T-cell differentiation. Nonetheless, the findings presented here strongly suggest that clinical studies on IFN- $\gamma$ in combination with PD1 checkpoint blockade are beneficial to human patients with PC.

\section{CONCLUSION}

We report that IFN- $\gamma$ suppresses the expression of tumor-derived CXCL8 and inhibits tumor-induced $\mathrm{CXCR}^{+} \mathrm{CD}^{+} 8^{+}$macrophage tumor trafficking by blocking the CXCL8-CXCR2 axis, thereby abolishing the local immunosuppressive effect of these cells. Combined with IFN- $\gamma$ treatment, PD1 checkpoint blockade showed significant antitumor effects in an established murine PC model (figure 6). Human PC also produces high levels of CXCL8 and patients with PC present elevated CXCR2 expression on peripheral and tumor-infiltrating $\mathrm{CD}^{+} 8^{+}$ macrophages, which are associated with advanced tumor stage and poor prognosis. These findings suggest that
IFN- $\gamma$ is an important agent to improve the potency of anti-PD1 therapy in patients with PC.

\section{Author affiliations}

${ }^{1}$ Department of Hepatobiliary and Pancreatic Surgery, Huzhou Central Hospital, Huzhou, China

${ }^{2}$ Key Laboratory of Biomedicine and Health, Hangzhou Normal University Hangzhou School of Medicine, Hangzhou, China

${ }^{3}$ Department of Surgery, Zhejiang University School of Medicine Sir Run Run Shaw Hospital, Hangzhou, China

${ }^{4}$ Key Laboratory of Molecular Medicine, Huzhou Central Hospital, Huzhou, China

Acknowledgements We thank Dr Ping Xie at the Department of Hepatobiliary Surgery, Huzhou Hospital, School of Medicine, Zhejiang University (Huzhou Central Hospital) for his assistance of providing blood and surgical samples of the patients with PC. We also thank Dr Hongfei Liu at the Key Laboratory of Biomedicine and Health, School of Medicine, Hangzhou Normal University for his assistance of immunofluorescence experiments.

Contributors MZ, GD, LC and QY developed the original hypothesis and designed the study. LH, HH, GC, TS, XX performed the experiments. XS, NL and QW performed the data analysis and provided statistical interpretation. MZ and LH wrote the manuscript. All authors read and approved the final manuscript.

Funding This work was supported by the Natural Science Foundation of Zhejiang Province (grant no. LGF18H160004 to MZ); the National Natural Science Foundation of China (grant no. 81772548 to LC); the Public Welfare Technology Application Research Project of Zhejiang Province (grant no. 2017 C33189 to QY); the Funds of Huzhou Municipal Science and Technology Bureau (grant no. 2018GY11 to MZ).

Competing interests None declared.

\section{Patient consent for publication Obtained.}

Ethics approval This study was approved by the ethics committee of Huzhou Hospital, School of Medicine, Zhejiang University (Huzhou Central Hospital) (HzhcLS2018-0807), China. Patients from Huzhou Central Hospital were obtained in our preclinical research with informed consent (ChiCTR1800017665).

Provenance and peer review Not commissioned; externally peer reviewed.

Data availability statement Data are available on reasonable request. The datasets used and analyzed during the present study are available from the corresponding author on reasonable request.

Open access This is an open access article distributed in accordance with the Creative Commons Attribution Non Commercial (CC BY-NC 4.0) license, which permits others to distribute, remix, adapt, build upon this work non-commercially, and license their derivative works on different terms, provided the original work is properly cited, appropriate credit is given, any changes made indicated, and the use is non-commercial. See http://creativecommons.org/licenses/by-nc/4.0/.

\section{ORCID iD}

Mingjie Zhang http://orcid.org/0000-0002-3193-7694

\section{REFERENCES}

1 Siegel RL, Miller KD, Jemal A. Cancer statistics, 2019. CA Cancer J Clin 2019;69:7-34.

2 Miller KD, Nogueira L, Mariotto AB, et al. Cancer treatment and survivorship statistics, 2019. CA Cancer J Clin 2019;69:363-85.

3 Martinez-Bosch N, Vinaixa J, Navarro P. Immune evasion in pancreatic cancer: from mechanisms to therapy. Cancers 2018;10:6.

4 Freeman GJ, Long AJ, Iwai Y, et al. Engagement of the PD-1 immunoinhibitory receptor by a novel B7 family member leads to negative regulation of lymphocyte activation. $J$ Exp Med 2000;192:1027-34.

5 Keir ME, Liang SC, Guleria I, et al. Tissue expression of PD-L1 mediates peripheral T cell tolerance. J Exp Med 2006;203:883-95.

6 Greenwald RJ, Freeman GJ, Sharpe AH. The B7 family revisited. Annu Rev Immunol 2005;23:515-48.

7 Dong H, Strome SE, Salomao DR, et al. Tumor-associated B7$\mathrm{H} 1$ promotes T-cell apoptosis: a potential mechanism of immune evasion. Nat Med 2002;8:793-800.

8 Birnbaum DJ, Finetti P, Lopresti A, et al. Prognostic value of PDL1 expression in pancreatic cancer. Oncotarget 2016;7:71198-210. 
9 Loos M, Giese NA, Kleeff J, et al. Clinical significance and regulation of the costimulatory molecule B7-H1 in pancreatic cancer. Cancer Lett 2008;268:98-109.

10 Nomi T, Sho M, Akahori T, et al. Clinical significance and therapeutic potential of the programmed death-1 ligand/programmed death-1 pathway in human pancreatic cancer. Clin Cancer Res 2007:13:2151-7.

11 Henriksen A, Dyhl-Polk A, Chen I, et al. Checkpoint inhibitors in pancreatic cancer. Cancer Treat Rev 2019;78:17-30.

12 Heusinkveld M, van der Burg SH. Identification and manipulation of tumor associated macrophages in human cancers. $J$ Transl Med 2011;9:216

13 Komohara Y, Takeya M. CAFs and TAMs: maestros of the tumour microenvironment. J Pathol 2017;241:313-5.

14 Lawrence T, Natoli G. Transcriptional regulation of macrophage polarization: enabling diversity with identity. Nat Rev Immunol 2011;11:750-61.

15 Mosser DM, Edwards JP. Exploring the full spectrum of macrophage activation. Nat Rev Immunol 2008;8:958-69.

16 Murray PJ, Wynn TA. Protective and pathogenic functions of macrophage subsets. Nat Rev Immunol 2011;11:723-37.

17 Bögels M, Braster R, Nijland PG, et al. Carcinoma origin dictates differential skewing of monocyte function. Oncoimmunology 2012;1:798-809.

18 Zhang Q, Green MD, Lang X, et al. Inhibition of ATM increases interferon signaling and sensitizes pancreatic cancer to immune checkpoint blockade therapy. Cancer Res 2019;79:3940-51.

19 Mantovani A, Marchesi F, Malesci A, et al. Tumour-associated macrophages as treatment targets in oncology. Nat Rev Clin Oncol 2017; 14:399-416.

20 Ostuni R, Kratochvill F, Murray PJ, et al. Macrophages and cancer: from mechanisms to therapeutic implications. Trends Immunol 2015;36:229-39.

21 Imai D, Yoshizumi T, Okano S, et al. IFN- $\gamma$ promotes epithelialmesenchymal transition and the expression of PD-L1 in pancreatic cancer. Journal of Surgical Research 2019;240:115-23.

22 Schroder K, Hertzog PJ, Ravasi T, et al. Interferon- $\gamma$. an overview of signals, mechanisms and functions. J Leukoc Biol 2004;75:163-89.

23 Baer C, Squadrito ML, Laoui D, et al. Suppression of microRNA activity amplifies IFN- $\gamma$-induced macrophage activation and promotes anti-tumour immunity. Nat Cell Biol 2016;18:790-802.

24 Zhang M, Ding G, Zhou L, et al. Interferon gamma inhibits CXCL8induced proliferation and migration of pancreatic cancer BxPC-3 cell line via a RhoGDI2/Rac1/NF-KB signaling pathway. J Interferon Cytokine Res 2018;38:413-22.

$25 \mathrm{Ha} \mathrm{H}$, Debnath B, Neamati N. Role of the CXCL8-CXCR1/2 axis in cancer and inflammatory diseases. Theranostics 2017;7:1543-88.

26 Litman-Zawadzka A, Łukaszewicz-Zając M, Gryko M, et al. Serum chemokine CXCL8 as a better biomarker for diagnosis and prediction of pancreatic cancer than its specific receptor CXCR2, C-reactive protein, and classic tumor markers Ca 19-9 and CEA. Pol Arch Intern Med 2018;128:524-31.

27 Chen L, Fan J, Chen $\mathrm{H}$, et al. The IL-8/CXCR1 axis is associated with cancer stem cell-like properties and correlates with clinical prognosis in human pancreatic cancer cases. Scientific Reports 2014;4.

28 ljichi H. Inhibition of CXCLs/CXCR2 axis in the tumor microenvironment might be a potent therapeutics for pancreatic cancer. Oncoimmunology 2012;1:569-71.

29 Pardoll DM. The blockade of immune checkpoints in cancer immunotherapy. Nat Rev Cancer 2012;12:252-64.
30 Topalian SL, Hodi FS, Brahmer JR, et al. Safety, activity, and immune correlates of anti-PD-1 antibody in cancer. N Engl J Med 2012;366:2443-54

31 Brahmer JR, Tykodi SS, Chow LQM, et al. Safety and activity of anti-PD-L1 antibody in patients with advanced cancer. N Engl J Med 2012;366:2455-65.

32 Feig C, Gopinathan A, Neesse A, et al. The pancreas cancer microenvironment. Clin Cancer Res 2012;18:4266-76.

$33 \mathrm{Li}$ X, Shao C, Shi Y, et al. Lessons learned from the blockade of immune checkpoints in cancer immunotherapy. $J$ Hematol Oncol 2018;11.

34 Chiaravalli M, Reni M, O'Reilly EM. Pancreatic ductal adenocarcinoma: state-of-the-art 2017 and new therapeutic strategies. Cancer Treat Rev 2017;60:32-43.

35 Xu W, Liu K, Chen M, et al. Immunotherapy for hepatocellular carcinoma: recent advances and future perspectives. Ther Adv Med Oncol 2019;11:1758835919862692.

$36 \mathrm{Xu}$ J, Zhang Y, Jia R, et al. Anti-Pd-1 antibody SHR-1210 combined with apatinib for advanced hepatocellular carcinoma, gastric, or esophagogastric junction cancer: an open-label, dose escalation and expansion study. Clin Cancer Res 2019;25:515-23.

37 Hodi FS, Chiarion-Sileni V, Gonzalez R, et al. Nivolumab plus ipilimumab or nivolumab alone versus ipilimumab alone in advanced melanoma (CheckMate 067): 4-year outcomes of a multicentre, randomised, phase 3 trial. Lancet Oncol 2018;19:1480-92.

38 Lemos H, Huang L, Prendergast GC, et al. Immune control by amino acid catabolism during tumorigenesis and therapy. Nat Rev Cancer 2019:19:162-75.

39 Lechner M, Lirk P, Rieder J. Inducible nitric oxide synthase (iNOS) in tumor biology: the two sides of the same coin. Semin Cancer Biol 2005:15:277-89.

40 Trellakis S, Bruderek K, Hütte J, et al. Granulocytic myeloid-derived suppressor cells are cryosensitive and their frequency does not correlate with serum concentrations of colony-stimulating factors in head and neck cancer. Innate Immun 2013;19:328-36.

41 Fan X, Patera AC, Pong-Kennedy A, et al. Murine CXCR1 is a functional receptor for GCP-2/CXCL6 and interleukin-8/CXCL8. J Biol Chem 2007;282:11658-66.

42 Chawla-Sarkar M, Lindner DJ, Liu Y-F, et al. Apoptosis and interferons: role of interferon-stimulated genes as mediators of apoptosis. Apoptosis 2003;8:237-49.

43 Miller CHT, Maher SG, Young HA. Clinical use of interferon-gamma. Ann N Y Acad Sci 2009;1182:69-79.

$44 \mathrm{Hu} X$, Ivashkiv LB. Cross-regulation of signaling pathways by interferon- $\gamma$ : implications for immune responses and autoimmune diseases. Immunity 2009;31:539-50.

45 Garcia-Diaz A, Shin DS, Moreno BH, et al. Interferon receptor signaling pathways regulating PD-L1 and PD-L2 expression. Cell Rep 2017;19:1189-201.

46 Benci JL, Johnson LR, Choa R, et al. Opposing functions of interferon coordinate adaptive and innate immune responses to cancer immune checkpoint blockade. Cell 2019;178:933-48.

47 Castro F, Cardoso AP, Gonçalves RM, et al. Interferon-gamma at the crossroads of tumor immune surveillance or evasion. Front Immunol 2018;9:847.

48 Yamaguchi R, Perkins G. Animal models for studying tumor microenvironment (TME) and resistance to lymphocytic infiltration. Cancer Biol Ther 2018;19:745-54. 